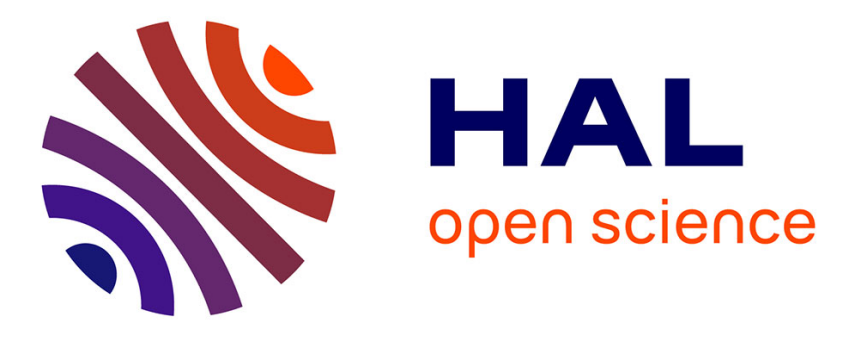

\title{
Environmentally-Safe Conditions for a \\ Palladium-Catalyzed Direct C3-Arylation with High Turn Over Frequency of Imidazo[1,2-b]pyridazines Using Aryl Bromides and Chlorides
}

Sabah Chikhi, Safia Djebbar, Jean-François Soulé, Henri Doucet

\section{- To cite this version:}

Sabah Chikhi, Safia Djebbar, Jean-François Soulé, Henri Doucet. Environmentally-Safe Conditions for a Palladium-Catalyzed Direct C3-Arylation with High Turn Over Frequency of Imidazo[1,2b]pyridazines Using Aryl Bromides and Chlorides. Chemistry , 2016, 11 (17), pp.2443-2452. 10.1002/asia.201600827. hal-01367228

\section{HAL Id: hal-01367228}

\section{https://hal-univ-rennes1.archives-ouvertes.fr/hal-01367228}

Submitted on 6 Feb 2017

HAL is a multi-disciplinary open access archive for the deposit and dissemination of scientific research documents, whether they are published or not. The documents may come from teaching and research institutions in France or abroad, or from public or private research centers.
L'archive ouverte pluridisciplinaire HAL, est destinée au dépôt et à la diffusion de documents scientifiques de niveau recherche, publiés ou non, émanant des établissements d'enseignement et de recherche français ou étrangers, des laboratoires publics ou privés. 


\title{
Environmentally-Safe Conditions for the Palladium-Catalyzed Direct C3-Arylation with High Turn Over Frequency of Imidazo[1,2-b]pyridazines Using Aryl Bromides and Chlorides
}

\author{
Sabah Chikhi, ${ }^{[a, b]}$ Safia Djebbar, ${ }^{[b]}$ Jean-François Soulé, ${ }^{*[a]}$ and Henri Doucet ${ }^{*[a]}$
}

\begin{abstract}
Phosphine-free $\mathrm{Pd}(\mathrm{OAc})_{2}$ was found to catalyze very efficiently the direct arylation of imidazo[1,2-b]pyridazine at $C 3$ position under a very low catalyst loading. The reaction can be performed employing as little as $0.1-0.05 \mathrm{~mol} \%$ catalyst with wide range of aryl bromides in very high TOFs and TONs. In addition, some electron-deficient aryl chlorides were also found to be suitable substrates. Moreover, 31 examples of those couplings were reported using green, safe and renewable solvents such as pentan1-ol, diethylcarbonate or cyclopentyl methyl ether, without loss of efficiency.
\end{abstract}

\section{Introduction}

In recent years, the Pd-catalyzed direct arylation of several heteroaromatics using aryl halides, via a $\mathrm{C}-\mathrm{H}$ bond activation, has become one of the most environmentally friendly methods for the C-C bond formation. ${ }^{[1]}$ Indeed, compared to other classical Pd-catalyzed reactions such as Stille, Suzuki or Negishi couplings, they do not require the preliminary synthesis of organometallic derivatives, and only $\mathrm{HX}$ associated to a base is generated as by-product. However, many challenge remains in order to get sustainable reaction conditions. For example, the major drawback of most of the described procedures is that they generally require $5-10 \mathrm{~mol} \%$ palladium catalyst associated to $10-20 \mathrm{~mol} \%$ of phosphine ligands. Since the discovery by de Vries and co-workers that only trace amount of phosphine-free catalyst $\mathrm{Pd}(\mathrm{OAc})_{2}$ (i.e., $0.1-0.01 \mathrm{~mol} \%$ ) are able to catalyze Heck and Suzuki reactions ${ }^{[2]}$-due to the soluble palladium(0) clusters formation at elevated temperature- this technology has been largely employed to promote the arylation of

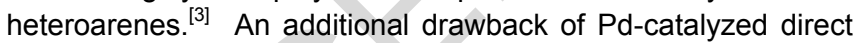
arylation in terms of green chemistry is that they often employ DMF or DMA as toxic or non-renewable solvents. According to the solvent selection guides published by chemical companies, DMF or DMA should be replaced by more sustainable solvents. ${ }^{[4]}$ Some reports demonstrated that $\mathrm{C}-\mathrm{H}$ bond arylation

[a] Ms. S. Chikhi, Dr. J.-F. Soulé, ${ }^{*}$ Dr. H. Doucet ${ }^{\star}$

Institution Institut des Sciences Chimiques de Rennes, UMR 6226 CNRS - Université de Rennes 1 Campus de Beaulieu, 35042 Campus de Beaulieu, 263 avenue du Général Leclerc 35042 Rennes Cedex (France)

E-mail: jean-francois.soule@univ-rennes1.fr, henri.doucet@univrennes1.fr

[b] Ms. S. Chikhi, Dr. S. Djebbar Laboratoire d'hydrométallurgie et chimie inorganique moléculaire Faculté de Chimie U.S.T.H.B. Bab-Ezzouar, Alger, Algeria.

Supporting information for this article is given via a link at the end of the document could be conducted in carbonates, ${ }^{[5]}$ such as diethylcarbonate (DEC), which is a polar, aprotic, nontoxic, and biodegradable solvent. ${ }^{[6]}$ Cyclopentyl methyl ether (CPME) is also a suitable alternative solvent for $\mathrm{C}-\mathrm{H}$ bond activation/functionalization. ${ }^{[7]}$ Highly polar and protic solvents, such as alcohols ${ }^{[8]}$ or water, ${ }^{[9]}$ have also been used in transition metal-catalyzed $\mathrm{C}-\mathrm{H}$ bond activation. However, the merging of low loading phosphine-free palladium catalyst conditions with eco-friendly or bio-sourced solvent remains a challenge, especially with aryl chlorides.

As a continuation of our researches on the development of environmentally-safe conditions for the Pd-catalyzed direct arylation, and based on our previous work on direct arylation of imidazo[1,2-a]pyridine, ${ }^{[3 \mathrm{~d}]}$ which displayed a very high reactivity, we turned our attention to the reactivity of imidazo[1,2b]pyridazine in direct arylation. Such heterocycles have potential applications in medicinal chemistry. As examples, the 3-arylimidazo[1,2-b]pyridazine scaffold displays selective inhibition of Mps1 (TTK) Kinase. ${ }^{[10]}$ In 2010, Berteina-Raboin, Guillaumet and co-workers described the first and unique example of direct arylations of 6-chloroimidazo[1,2-b]pyridazine with aryl bromides. ${ }^{[11]}$ They reported that the use of $10 \mathrm{~mol} \%$ $\mathrm{Pd}(\mathrm{OAc})_{2}$ associated to $20 \mathrm{~mol}^{2} \mathrm{PPh}_{3}$ in toluene promotes efficiently such couplings in good yields. Due to the important biological properties of 3-arylimidazo[1,2-b]pyridazines, the discovery of more environmentally-safe conditions for the direct coupling of imidazo[1,2-b]pyridazine derivatives with aryl bromides, especially using low loadings of a phosphine-free catalyst in renewable solvents, would be a considerable advantage for industrial applications.

\section{Results and Discussion}

Imidazo[1,2-b]pyridazine and 4-bromobenzonitrile were used as model substrates, and we choose our previously described optimized reaction conditions for the direct arylation of imidazo[1,2-a]pyridine (i.e., $\mathrm{Pd}(\mathrm{OAc})_{2}$ as catalyst, 2 equivalents of KOAC, in DMA at $150^{\circ} \mathrm{C}$ ) (Table 1). ${ }^{[3 \mathrm{~d}]}$ We found that the $\mathrm{C} 3$ arylated product 1 was obtained in very high yield using only 1 $\mathrm{mol} \%$ of $\mathrm{Pd}(\mathrm{OAc})_{2}$ without phosphine ligand (Table 1, entry 1). The TONs of the reaction were increased to 1940 and 8900 using 0.05 or $0.01 \mathrm{~mol} \%$ catalyst loadings, respectively (Table 1 , entries 2-4). In order to discover safer reaction conditions, we decided to evaluate greener solvents for this coupling using 0.05 mol\% of $\mathrm{Pd}(\mathrm{OAc})_{2}$. Pentan-1-ol, DEC, and CPME allowed the formation of desired product 1 in high yields (Table 1, entries 6 , 8 , and 10). As the association of palladium salts to pyridine derivatives as ligands provides in some cases very efficient catalysts for direct arylation reactions, ${ }^{[12]}$ we also performed a comparison study using 1,10-phenanthroline as ligand (Table 1, entries $5,7,9$ and 11). In all cases, the addition of $0.05 \mathrm{~mol} \%$ of 1,10 -phenanthroline to the reaction mixture did not allowed any 
yield improvement or change in the regioselectivity. We also performed the reaction at a lower temperature of $100^{\circ} \mathrm{C}$ (Table 1, entries 12-14). However, lower TONs were obtained whatever the employed solvent. As expected, in the absence of $\mathrm{Pd}(\mathrm{OAc})_{2}$ catalyst, no reaction occurred (Table 1, entry 15). It is important to note that all the reactions which employed 0.05 or $0.01 \mathrm{~mol} \%$ catalyst were performed using new Schlenk tubes and new stirring bars to avoid contamination by palladium residues.

Table 1. Influence of the Reaction Conditions

\begin{tabular}{|c|c|c|c|c|c|}
\hline Entry & $(\mathrm{x} \mathrm{mol} \%)$ & Solvent & $\begin{array}{l}\text { Conv. } \\
(\%)^{[a]}\end{array}$ & $\begin{array}{l}\text { Yield in } \\
\mathbf{1}(\%)^{[b]}\end{array}$ & TON \\
\hline 1 & 1 & DMA & 100 & 98 & 98 \\
\hline 2 & 0.5 & DMA & 100 & 98 & 196 \\
\hline 3 & 0.05 & DMA & 100 & 97 & 1940 \\
\hline 4 & 0.01 & DMA & 92 & 89 & 8900 \\
\hline $5^{[\mathrm{cc}]}$ & 0.05 & DMA & 69 & 65 & 1300 \\
\hline 6 & 0.05 & pentan-1-ol & 100 & 97 & 1940 \\
\hline $7^{[c]}$ & 0.05 & pentan-1-ol & 0 & 0 & 0 \\
\hline 8 & 0.05 & DEC & 100 & 90 & 1900 \\
\hline $9^{[c]}$ & 0.05 & DEC & 78 & 78 & 1560 \\
\hline 10 & 0.05 & CPME & 100 & 96 & 1920 \\
\hline $11^{[\mathrm{c}]}$ & 0.05 & CPME & 38 & 6 & 720 \\
\hline $12^{[\mathrm{d}]}$ & 0.5 & DMA & 67 & 66 & 132 \\
\hline $13^{[\mathrm{d}]}$ & 0.5 & pentan-1-ol & 37 & 37 & 74 \\
\hline $14^{[\mathrm{d}]}$ & 0.5 & DEC & 71 & 68 & 136 \\
\hline 15 & 0 & DMA & & - & - \\
\hline
\end{tabular}

[a] Based on 4-bromobenzonitrile consumption using GC-MS analysis with dodecane as internal standard. [b] Isolated yield. [c] In the presence of $0.05 \mathrm{~mol} \%$ of 1,10-phenanthroline. [d] Reaction performed at $100^{\circ} \mathrm{C}$

As full consumption of 4-bromobenzonitrile was observed in all cases in the presence of $0.05 \mathrm{~mol} \%$ catalyst using DMA, DEC, CPME, or pentan-1-ol, we decided to plot a TON versus time of this model reaction with the different solvents (Figure 1a). Remarkably, all the solvents were compatible under our standard catalytic conditions $\left(0.05 \mathrm{~mol} \% \mathrm{Pd}(\mathrm{OAc})_{2}, \mathrm{KOAc}(2\right.$ equiv.) at $150{ }^{\circ} \mathrm{C}$ ), but clear differences among them were evident. The reaction performed in CPME was the slowest for the formation of arylated product $1\left(\right.$ TOF $\left.^{10 \mathrm{~min}}=3000 \mathrm{~h}^{-1}\right)$, albeit the reaction was complete after $2 \mathrm{~h}$. Pentan-1-ol displayed a higher efficiency, as a TOF ${ }^{10 \mathrm{~min}}$ of $5040 \mathrm{~h}^{-1}$ was obtained after 10 minutes. The reaction performed in DEC indicated a TOF ${ }^{10 \mathrm{~min}}$ of $7560 \mathrm{~h}^{-1}$ after 10 minutes. The highest TOF after 10 minutes was obtained when the reaction was performed in DMA, with complete conversion after only 30 minutes and a TOF ${ }^{10 \mathrm{~min}}$ of $10320 \mathrm{~h}^{-1}$ after 10 minutes. Similar experiments were conducted with electron-rich 4-bromoanisole (Figure 1b). With all solvents conditions, the desired C3-arylated imidazo[1,2-b]pyridazine 2 was obtained, but again marked differences were observed. Again, the reaction in DMA was the most efficient to deliver the coupling product 2, although a lower TOF ${ }^{10 m i n}$ of $2760 \mathrm{~h}^{-1}$ than with 4-bromobenzonitrile was obtained. The reaction performed in pentan-1-ol also displayed a high TOF $\left(\operatorname{TOF}^{10 \mathrm{~min}}=1920 \mathrm{~h}^{-1}\right)$; whereas, in DEC or CPME the reactions were not complete after $5 \mathrm{~h}$, with conversions of $80 \%$ and $42 \%$, respectively and lower TOFs ${ }^{10 \mathrm{~min}}$ of $960 \mathrm{~h}^{-1}$ and $480 \mathrm{~h}^{-1}$

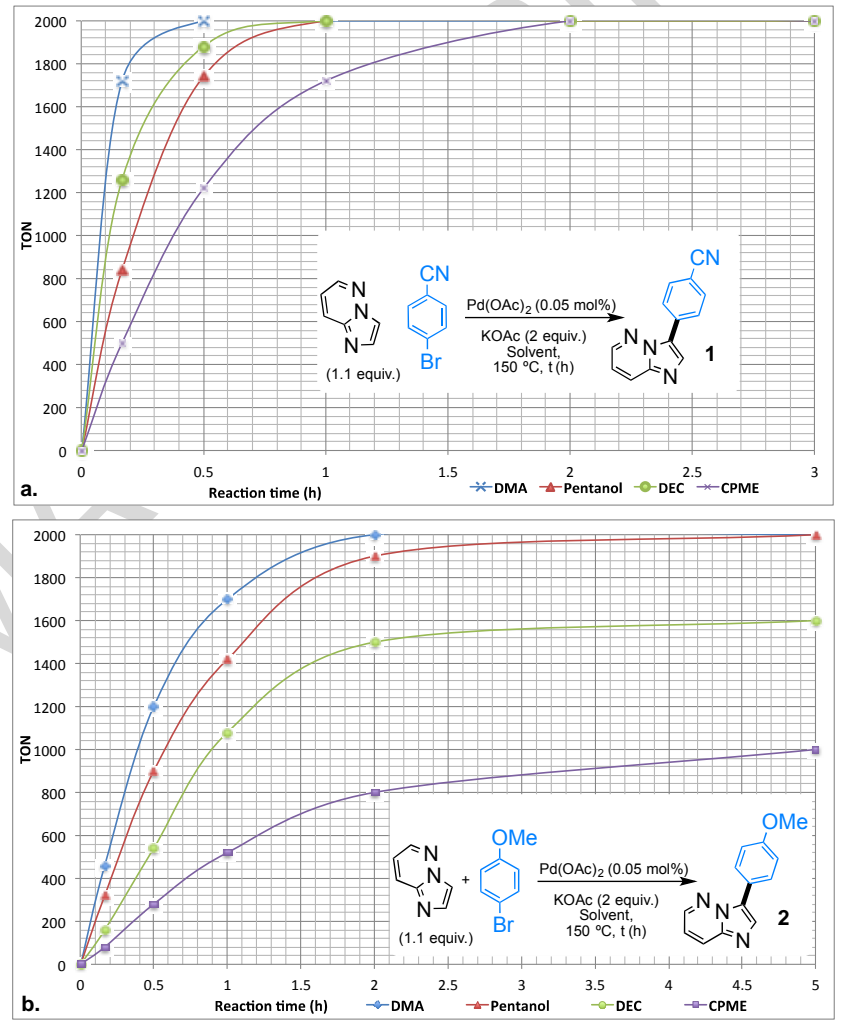

Figure 1. TON vs Time of Pd-Catalyzed Coupling Reactions between Imidazo[1,2-b]pyridazine in Different Solvents: a) with 4-bromobenzonitrile or b) with 4-bromoanisole.

With these catalytic systems in hands, we decided to investigate the scope of aryl bromides in the Pd-catalyzed direct C3arylation of imidazo[1,2-b]pyridazine using a low loading of phosphine-free palladium catalyst (i.e., $\mathrm{Pd}(\mathrm{OAc})_{2} 0.05 \mathrm{~mol} \%$ ) (Scheme 1). As seen above, 4-bromoanisole reacted nicely with imidazo[1,2-b]pyridazine to give the arylated heterocycle 2 in $79 \%$ isolated yield. Bromobenzenes para-substituted by nitro, formyl or acetyl groups gave the C3-arylated products 3-5 in high yields in both DMA and pentan-1-ol. Ethyl 4bromobenzoate was also tolerated by the optimized reaction conditions to afford 6 in $91 \%$ yield. Using solvent free-conditions, a slightly lower yield of $51 \%$ was obtained. Chloro or fluoro substituents at para-position of bromobenzene have almost no 
effect on the reaction, as the coupling products 7 and 8 were isolated in $89 \%$ and $93 \%$ yields, respectively. The reaction with 4-bromotoluene as coupling partner smoothly proceeded in DMA or in pentan-1-ol to afford the coupling product 9 in $82 \%$ or $78 \%$ yield. Interestingly, 4-iodoaniline, which is a very electron-rich substrate, was also tolerated to afford the heterocycle 10 in 91\%; whereas, 4-bromoaniline was unreactive. It is important to note that the direct arylation proceeded faster than amination reaction. ${ }^{[13]}$ The reaction performed in pentan-1-ol instead of DMA led to a lower yield of $58 \%$ in $\mathbf{1 0}$. Meta-substituted aryl bromides reacted with imidazo[1,2-b]pyridazine in the same line than the para-substituted ones. As examples, methyl 3bromobenzoate and 3-bromoacetophenone gave the desired coupling products $\mathbf{1 1}$ and $\mathbf{1 2}$ in excellent yields in DMA or pentan-1-ol. 1,3-Dibromobenzene could be used for the selective mono-heteroarylation, affording 3-(3bromophenyl)imidazo[1,2-b]pyridazine 13 , in which one $\mathrm{C}-\mathrm{Br}$ bond remained untouched and could be used in further $\mathrm{Pd}$ catalyzed reactions. 3-lodoaniline was nicely coupled with imidazo[1,2-b]pyridazine in DMA to afford 14 in $82 \%$ yield without amination as side-reaction. A very electron-deficient aryl bromide, such as 3,5-bis(trifluoromethyl)bromobenzene, was also tolerated by the optimized conditions to afford the arylated heterocycle 15 in $90 \%$ yield. The reaction seems to be insensitive to the steric hindrance. Indeed, aryl bromides bearing 2-cyano, 2-formyl or 2-trifluoromethyl substituents nicely reacted to afford $\mathbf{1 6 - 1 8}$ in good yields. Notably, such orthosubstituted aryl bromides could be coupled in pentan-1-ol without loss of efficiency. From 1- bromonaphthalene or 1bromopyrene, the C3 polycyclic hydrocarbons substituted imidazo[1,2-b]pyridazine 19 and 20 were isolated in $74 \%$ and $79 \%$ yields, respectively. Heteroaryl bromides, such as 3bromoquinoline, 3-bromopyridine and 5-bromopyrimidine were also successfully coupled to give the heterocycle dyads 21-23 in $73-94 \%$ yields. Again, these reactions could be performed in pentan-1-ol or CPME instead of DMA. We also demonstrated that the coupling products could be isolated by recrystallization in cyclohexane after simple removal of DMA (e.g., 3, 5, 15, and 18 in scheme 1), instead of silica gel column chromatography, affording a very environmentally-attractive procedure for the synthesis of such compounds.

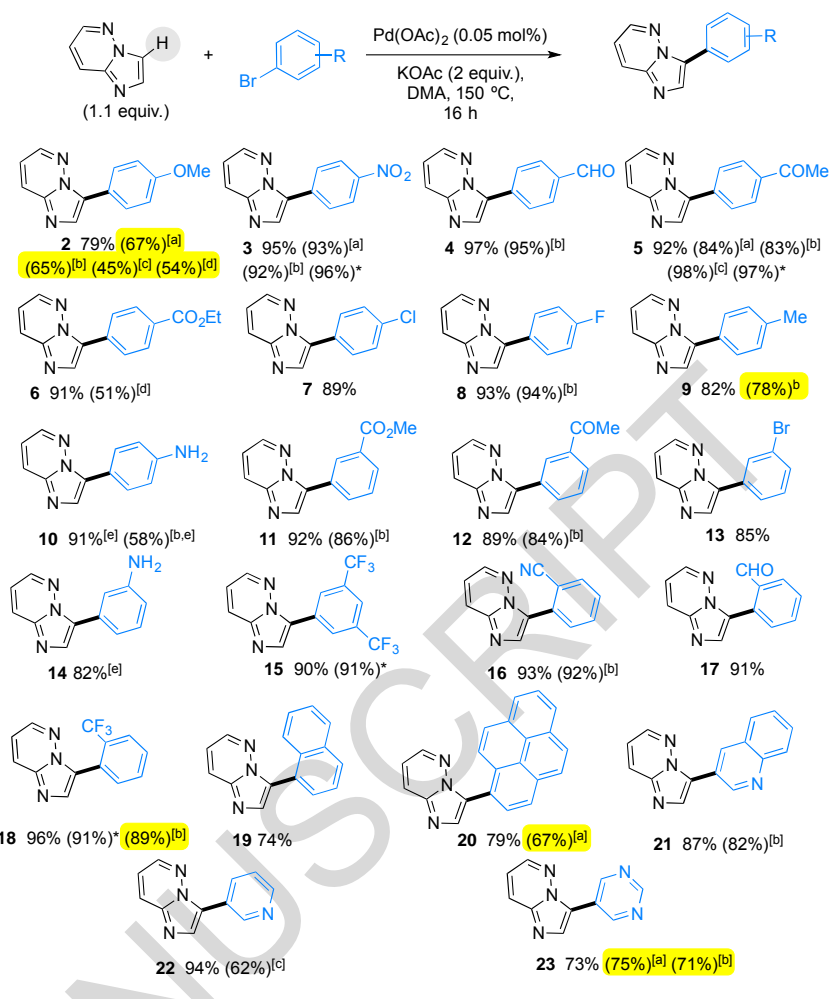

Scheme 1. Scope of Aryl Bromides in Pd-Catalyzed Direct Arylation of Imidazo[1,2-b]pyridazine. [a] Reaction performed in DEC instead of DMA. [b] Reaction performed in pentan-1-ol instead of DMA. [c] Reaction performed in CPME instead of DMA. [d] Reaction performed under neat conditions. [e] Reaction performed from $\mathrm{Arl}$ instead of $\mathrm{ArBr}$. * Reaction performed on 10 mmol scale; after removal of DMA under vacuo, the product was isolated by simple recrystallization in cyclohexane.

Having successfully coupled a wide range of aryl bromides with imidazo[1,2-b]pyridazine, we next investigated the efficiently of this simple catalytic system -namely, phosphine-free $\mathrm{Pd}(\mathrm{OAc})_{2}$ in the presence of KOAc- to promote direct arylation using aryl chlorides. Rare use has been made of (hetero)aryl chlorides in $\mathrm{Pd}$-catalyzed direct arylations, despite the fact that among aryl halides, aryl chlorides exhibit the wider diversity of available compounds at an affordable cost. In most cases, expensive ligands or catalytic systems should be used to overcome their poor reactivity in Pd-catalyzed direct arylation. ${ }^{[14]}$ Only a few reports focused on the use of phosphine-free palladium catalyts. $^{[3 e, 15]}$ First, we decided to evaluate several solvents for this coupling using 4-chlorobenzonitrile as aryl source in the presence of $0.1 \mathrm{~mol} \%$ of $\mathrm{Pd}(\mathrm{OAc})_{2}$ and 2 equivalents of $\mathrm{KOAc}$ as base without any additive (Figure 2). Interestingly, a full conversion was observed in DMA after $2 \mathrm{~h}$ with a TOF ${ }^{30 \mathrm{~min}}$ of $840 \mathrm{~h}^{-1}$ after 30 minutes. The reaction was more sluggish in other solvents such as pentan-1-ol or DEC, but full conversions could be reached after $6 \mathrm{~h}$. It is important to note that DEC has been previously used as eco-friendly solvent in $\mathrm{Pd}$-catalyzed direct arylation of several heteroarenes in the presence of aryl chlorides as aryl source, but diphosphine ligands were added to the reaction mixture. ${ }^{[16]}$ To the best of our knowledge, this is one of the rare examples of merging phosphine-free palladium catalyst with eco-friendly or bio-sourced solvents for the direct 
arylation of heteroarenes using aryl chlorides as aryl source. On the contrary, CPME as solvent was almost ineffective for this transformation.

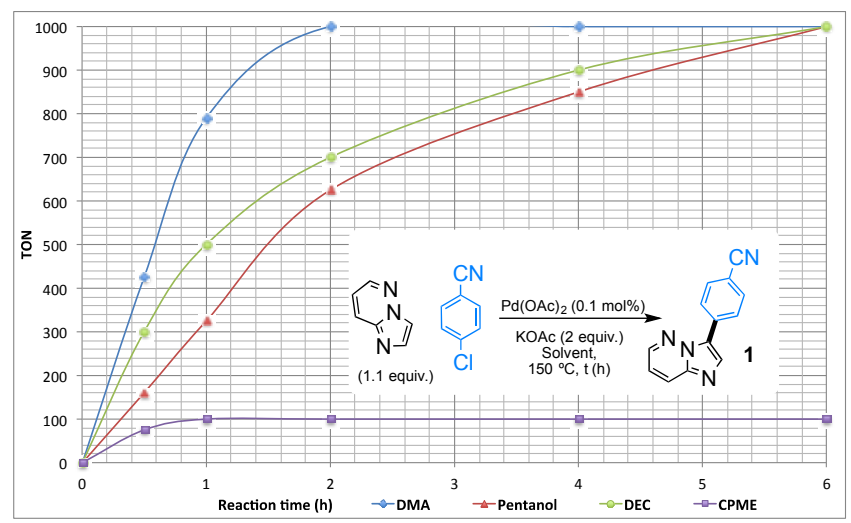

Figure 2. TON vs Time of Pd-Catalyzed Coupling Reaction Between Imidazo[1,2-b]pyridazine with 4-Chloronitrobenzene in Different Solvents.

Having found effective conditions for the use of activated aryl chlorides, namely, $0.1 \mathrm{~mol} \%$ of $\mathrm{Pd}(\mathrm{OAc})_{2}$ in the presence of 2 equivalents of KOAc in DMA, we turned our attention to the aryl chloride scope for these $\mathrm{Pd}$-catalyzed $\mathrm{C}-\mathrm{H}$ bond arylations. Chlorobenzenes substituted by electron-withdrawing groups at para-position such as $\mathrm{CN}, \mathrm{NO}_{2}, \mathrm{CF}_{3}, \mathrm{CO}_{2} \mathrm{Me}$ allowed the synthesis of the 3-arylated imidazo[1,2-b]pyridazines 1, 3, 24 and 25 in $69-92 \%$ yields. From 3-chlorobenzonitrile and 3chloronitrobenzene, the coupling products 26 and 27 were isolated in excellent yields. Under the same conditions, the reaction proceeded smoothly in the presence of orthosubstituted aryl chlorides. For examples, from 2chloronitrobenzene or 2-chloro-5-nitrobenzonitrile, the C3 arylated products $\mathbf{2 8}$ and $\mathbf{2 9}$ were obtained in $65 \%$ and $72 \%$ yields, respectively. Some of these couplings were also successfully performed in DEC and pentan-1-ol as alternative sustainable solvents. However, aryl chlorides bearing an electron-donating substituent (e.g., OMe or Me) were unreactive under these phosphine-free conditions.

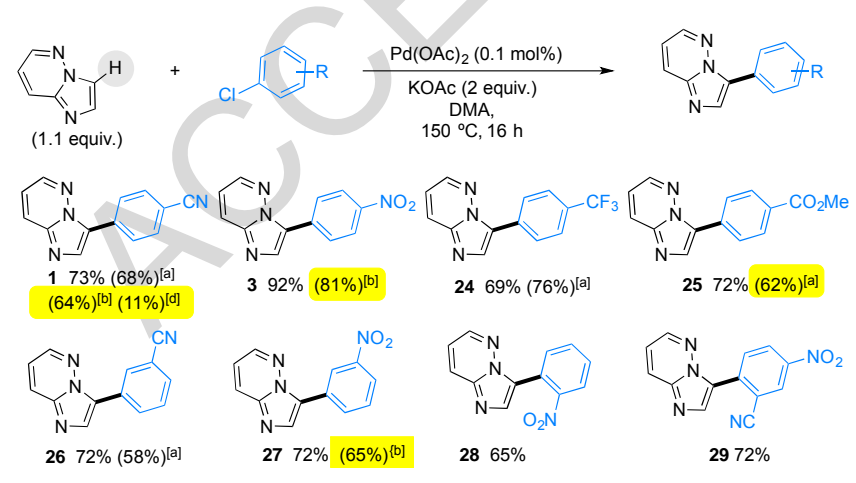

Scheme 2. Scope of Aryl Chlorides in Pd-Catalyzed Direct Arylation of Imidazo[1,2-b]pyridazine. [a] Reaction performed in DEC instead of DMA. [b] Reaction performed in pentan-1-ol instead of DMA. [c] Reaction performed under neat conditions.
In addition, we investigated the Pd-catalyzed direct arylation of 6-chloroimidazo[1,2-b]pyridazine in environmentally-safe conditions, namely $0.1 \mathrm{~mol} \% \mathrm{Pd}(\mathrm{OAc})_{2}$ associated to KOAc as base in pentan-1-ol. Electron-poor para-substituted aryl bromides, such as 4-bromonitrobenzene and 4bromobenzaldehyde, gave the coupling products $\mathbf{3 0}$ and $\mathbf{3 1}$ in $78 \%$ and $82 \%$ yields, respectively. Notably, the reaction could also be performed from 4-chloronitrobenzene to afford $\mathbf{3 0}$ in a similar yield, albeit DMA should be used as solvent. The reaction with 1,4-dibromobenzene selectively gave the monoarylated product 32 in $75 \%$ yield, which contains a $\mathrm{C}-\mathrm{Br}$ bond allowing further transformations. A second direct arylation from 32 via the activation of its $\mathrm{C}-\mathrm{Br}$ bond was not observed. This poor reactivity is due to a slow oxidative addition of $\mathbf{3 2}$ to palladium owing the electron-donor character of the first introduced heteroarene. Bulky aryl bromides, such as methyl 2bromobenzoate and 1-bromonaphthalene, were also tolerated by the optimized environmentally-safe conditions to afford the products 33 and 34 in $59 \%$ and $76 \%$ yields, respectively. Heteroaryl bromides have also been successfully coupled with 6-chloroimidazo[1,2-b]pyridazine to offer 35 and $\mathbf{3 6}$ in good yields. In all cases, no cleavage of the imidazopyridazine $\mathrm{C}-\mathrm{Cl}$ bond was observed. This chemoselectivity might be explained by the poor reactivity of electron-rich aryl chlorides under phosphine-free palladium conditions.

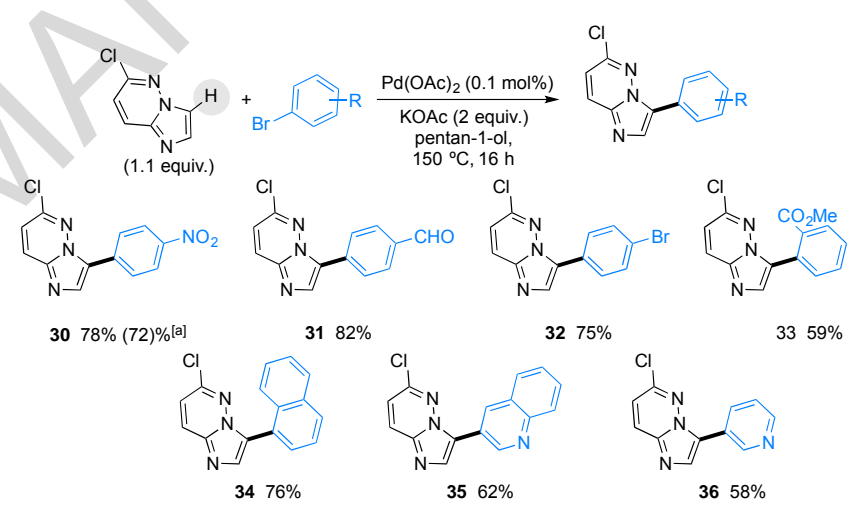

Scheme 3. Scope of Aryl Bromides and Chlorides in Pd-Catalyzed Direct Arylation of 6 -Chloroimidazo[1,2-b]pyridazine. [a] The reaction was performed from 4-chloronitrobenzene instead of 4-bromonitrobenzene, and DMA was used as solvent instead of pentan-1-ol.

Finally, we investigated the synthesis of 2,3-diarylimidazo[1,2b]pyridazine derivatives (Scheme 4). From 3-(4fluorophenyl)imidazo[1,2-b]pyridazine $\mathbf{8}$, we attempted to functionalize directly the $\mathrm{C} 2$ position through $\mathrm{Pd}$-catalyzed direct arylation. However, this synthetic scheme failed. Hence, we considered a two steps synthesis involving the preparation of 2bromo-3-(4-fluorophenyl)imidazo[1,2-b]pyridazine 37 intermediate. From aryl bromide derivative 37, classical Suzuki reaction conditions using phenylboronic acid or 3-thienylboronic acid as coupling partners allowed the formation of 2,3diarylimidazo[1,2-b]pyridazines $\mathbf{3 8}$ and 39 in $86 \%$ and $89 \%$ yields, respectively. 


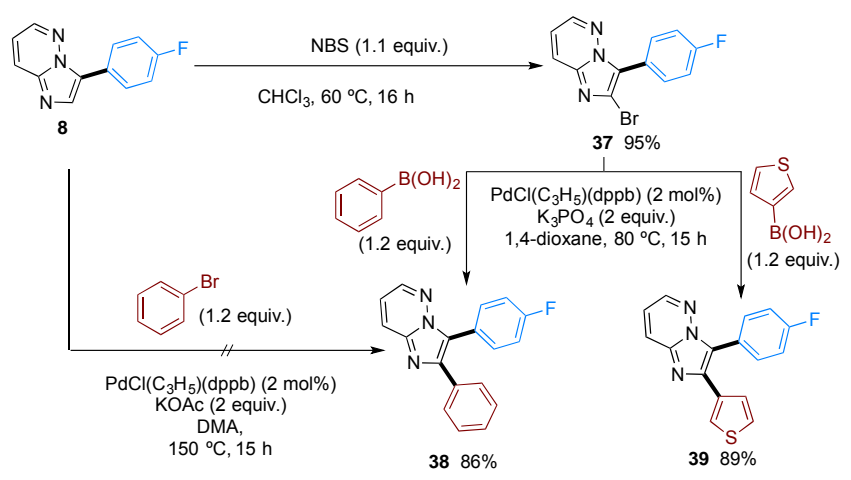

Scheme 4. Examples of Synthesis of 2,3-Diarylimidazo[1,2-b]pyridazine Derivatives.

\section{Conclusions}

In summary, we have developed environmentally-safe conditions for the direct C3 arylation of imidazo[1,2$b$ pyridazines. We found that the reaction proceed with very high TOFs and TONs using as little as $0.1-0.05 \mathrm{~mol} \%$ of phosphine-free $\mathrm{Pd}(\mathrm{OAc})_{2}$ as catalyst precursor. With this procedure, there is no need to eliminate phosphine residues at the end of the reaction. In addition, for several examples (i.e., 31 compounds over 36 ), we demonstrated that DMA, which is a toxic solvent, can be successfully substituted by eco-friendly or bio-resourced solvents, such as pentan-1-ol and diethylcarbonate, providing a greener approach to the synthesis of 3-aryl imidazo[1,2-b]pyridazines. The reaction conditions allowed the use of a wide variety of substituents on the aryl bromide such as ester, acetyl, formyl, propionyl, benzoyl, nitro, nitrile, bromo, chloro, fluoro, methoxy and amino. In addition, heteroaryl bromides could also employed. Moreover, some electron-deficient aryl chlorides, which are very attractive substrates in terms of atom-economy and cost, have been used instead of aryl bromides with comparable yields.

\section{Experimental Section}

All reactions were carried out under argon atmosphere with standard Schlenk techniques. 1,4-Dioxane, DMA, diethylcarnonate and pentan-1ol were purchased from Acros Organics and were not purified before use. ${ }^{1} \mathrm{H}$ NMR spectra were recorded on Bruker GPX (400 MHz) spectrometer. Chemical shifts $(\delta)$ were reported in parts per million relative to residual DMSO (2.50 ppm (quintet, $J_{H D}=1.9 \mathrm{~Hz}$ ) ppm for ${ }^{1} \mathrm{H} ; 39.52 \mathrm{ppm}$ for ${ }^{13} \mathrm{C}$ ), constants were reported in Hertz. ${ }^{1} \mathrm{H}$ NMR assignment abbreviations were the following: singlet $(\mathrm{s})$, doublet $(\mathrm{d})$, triplet $(\mathrm{t})$, quartet $(\mathrm{q})$, doublet of doublets (dd), doublet of triplets (dt), and multiplet $(\mathrm{m}) .{ }^{13} \mathrm{C} N M R$ spectra were recorded at $100 \mathrm{MHz}$ on the same spectrometer and reported in ppm. All reagents were weighed and handled in air.

Preparation of the $\mathrm{PdCl}(\mathrm{dppb})\left(\mathrm{C}_{3} \mathrm{H}_{5}\right)$ catalyst: ${ }^{[17]}$ An oven-dried $40-\mathrm{mL}$ Schlenk tube equipped with a magnetic stirring bar under argon atmosphere, was charged with $\left[\mathrm{Pd}\left(\mathrm{C}_{3} \mathrm{H}_{5}\right) \mathrm{Cl}\right]_{2}(182 \mathrm{mg}, 0.5 \mathrm{mmol})$ and $\mathrm{dppb}(426 \mathrm{mg}, 1 \mathrm{mmol}) .10 \mathrm{~mL}$ of anhydrous dichloromethane were added, then the solution was stirred at room temperature for twenty minutes. The solvent was removed in vacuum. The yellow powder was used without purification. ${ }^{31} \mathrm{P}$ NMR $\left(81 \mathrm{MHz}, \mathrm{CDCl}_{3}\right) \delta(\mathrm{ppm})=19.3$ (s).
General Procedure. As a typical experiment, the reaction of the aryl halides $(1 \mathrm{mmol})$, imidazo[1,2-b]pyridazine $(0.131 \mathrm{~g}, 1.1 \mathrm{mmol})$ or 6 chloroimidazo[1,2-b]pyridazine $(0.169 \mathrm{~g}, 1.1 \mathrm{mmol})$, and KOAc $(0.196 \mathrm{~g}$, $2 \mathrm{mmol}$ ) at $150{ }^{\circ} \mathrm{C}$ over $16 \mathrm{~h}$ in $4 \mathrm{~mL}$ of a freshly prepared solution of solvent (see tables or schemes) which contains the appropriate amount of $\mathrm{Pd}(\mathrm{OAc})_{2}$ (see preparation of this solution below), under argon affords the coupling product after evaporation of the solvent and purification on silica gel or recrystallization in cyclohexane.

A solution of $\mathrm{Pd}(\mathrm{OAc})_{2}$ was freshly prepared using $2.8 \mathrm{mg}$ of $\mathrm{Pd}(\mathrm{OAc})_{2}$ $(0.0125 \mathrm{mmol})$ and $100 \mathrm{~mL}$ of the appropriate solvent (DMA, pentan-1-ol, DEC, or CPME) under argon."

4-(Imidazo[1,2-b]pyridazin-3-yl)benzonitrile (1): Following the general procedure using 4-bromobenzonitrile $(0.182 \mathrm{~g}, 1 \mathrm{mmol})$ and imidazo[1,2b]pyridazine $(0.131 \mathrm{~g}, 1.1 \mathrm{mmol})$, the residue was purified by flash chromatography on silica gel (pentane- $\mathrm{Et}_{2} \mathrm{O}, 70: 30$ ) to afford the desired compound $1(0.216 \mathrm{~g}, 98 \%)$ as a yellow solid $\left(\mathrm{mp}=156-158{ }^{\circ} \mathrm{C}\right) .{ }^{1} \mathrm{H}$ NMR (400 MHz, $d_{6}$-DMSO) $\delta(p p m) 8.71$ (dd, $J=1.6$ and $4.5 \mathrm{~Hz}, 1 \mathrm{H}, \mathrm{H} 6$ ), $8.49(\mathrm{~s}, 1 \mathrm{H}, \mathrm{H} 2), 8.42(\mathrm{~d}, \mathrm{~J}=8.5 \mathrm{~Hz}, 2 \mathrm{H}), 8.27$ (dd, $\mathrm{J}=1.6$ and $9.2 \mathrm{~Hz}$, $1 \mathrm{H}, \mathrm{H} 8), 7.97$ (d, J = 8.5 Hz, 2H), 7.38 (dd, $\mathrm{J}=4.5$ and $9.2 \mathrm{~Hz}, 1 \mathrm{H}, \mathrm{H} 7$ ). ${ }^{13} \mathrm{C}$ NMR (100 MHz, $d_{6}$-DMSO) $\delta(\mathrm{ppm}) 144.3,140.9,134.7,132.9$, 132.6, 126.3, 126.1, 125.5, 118.9, 118.1, 109.5. Elemental analysis: calcd (\%) for $\mathrm{C}_{13} \mathrm{H}_{8} \mathrm{~N}_{4}$ (220.23): C 70.90, H 3.66; found: C 71.19, H 3.86.

3-(4-Methoxyphenyl)imidazo[1,2-b]pyridazine (2): Following the general procedure using 4-bromoanisole $(0.187 \mathrm{~g}, 1 \mathrm{mmol})$ and imidazo[1,2-b]pyridazine $(0.131 \mathrm{~g}, 1.1 \mathrm{mmol})$, the residue was purified by flash chromatography on silica gel (pentane- $\mathrm{Et}_{2} \mathrm{O}, 70: 30$ ) to afford the desired compound $2(0.178 \mathrm{~g}, 79 \%)$ as a yellow solid $\left(\mathrm{mp}=75-79^{\circ} \mathrm{C}\right)$. ${ }^{1} \mathrm{H}$ NMR (400 MHz, $d_{6}$-DMSO) $\delta(p p m) 8.59$ (dd, $J=1.6$ and $4.4 \mathrm{~Hz}, 1 \mathrm{H}$, $\mathrm{H} 6), 8.20-8.15(\mathrm{~m}, 2 \mathrm{H}, \mathrm{H} 2$ and $\mathrm{H} 8), 8.05(\mathrm{~d}, \mathrm{~J}=8.9 \mathrm{~Hz}, 2 \mathrm{H}), 7.23(\mathrm{dd}, \mathrm{J}=$ 4.4 and $9.2 \mathrm{~Hz}, 1 \mathrm{H}, \mathrm{H} 7), 7.06(\mathrm{~d}, \mathrm{~J}=8.9 \mathrm{~Hz}, 2 \mathrm{H}), 3.81(\mathrm{~s}, 3 \mathrm{H}) .{ }^{13} \mathrm{C}$ NMR (100 MHz, $d_{6}$-DMSO) $\delta(p p m) ~ 158.8,143.7,139.3,131.8,127.8,127.3$, $125.9,120.9,116.5,114.1,55.1$. Elemental analysis: calcd (\%) for $\mathrm{C}_{13} \mathrm{H}_{11} \mathrm{~N}_{3} \mathrm{O}$ (225.25) C 69.32, H 4.92; found: C 69.58, H 5.17.

3-(4-Nitrophenyl)imidazo[1,2-b]pyridazine (3): Following the general procedure using 4-bromonitrobenzene $(0.200 \mathrm{~g}, 1 \mathrm{mmol})$ and imidazo[1,2-b]pyridazine $(0.131 \mathrm{~g}, 1.1 \mathrm{mmol})$, the residue was purified by flash chromatography on silica gel (pentane- $\mathrm{Et}_{2} \mathrm{O}, 55: 45$ ) to afford the desired compound $3(0.228 \mathrm{~g}, 95 \%)$ as a yellow solid $\left(\mathrm{mp}=207-210^{\circ} \mathrm{C}\right)$. ${ }^{1} \mathrm{H}$ NMR (400 MHz, $d_{6}$-DMSO) $\delta(\mathrm{ppm}) 8.76(\mathrm{dd}, \mathrm{J}=1.7$ and $4.4 \mathrm{~Hz}, 1 \mathrm{H}$, $\mathrm{H} 6), 8.57(\mathrm{~s}, 1 \mathrm{H}, \mathrm{H} 2), 8.52(\mathrm{~d}, \mathrm{~J}=8.9 \mathrm{~Hz}, 2 \mathrm{H}), 8.37(\mathrm{~d}, \mathrm{~J}=8.9 \mathrm{~Hz}, 2 \mathrm{H})$, 8.31 (dd, $J=1.7$ and $9.1 \mathrm{~Hz}, 1 \mathrm{H}, \mathrm{H} 8), 7.42(\mathrm{dd}, \mathrm{J}=4.4$ and $9.1 \mathrm{~Hz}, 1 \mathrm{H}$, H7). ${ }^{13} \mathrm{C}$ NMR (100 MHz, $d_{6}$-DMSO) $\delta$ (ppm) 145.8, 144.4, 141.2, 135.3, $134.9,126.5,126.4,126.3,124.1,118.5$. Elemental analysis: calcd (\%) for $\mathrm{C}_{12} \mathrm{H}_{8} \mathrm{~N}_{4} \mathrm{O}_{2}$ (240.22): C 60.00, H 3.36; found: C 59.79, H 3.48.

4-(Imidazo[1,2-b]pyridazin-3-yl)benzaldehyde (4): Following the general procedure using 4-bromobenzaldehyde $(0.183 \mathrm{~g}, 1 \mathrm{mmol})$ and imidazo[1,2-b]pyridazine $(0.131 \mathrm{~g}, 1.1 \mathrm{mmol})$, the residue was purified by flash chromatography on silica gel (pentane- $\mathrm{Et}_{2} \mathrm{O}, 65: 35$ ) to afford the desired compound $4(0.217 \mathrm{~g}, 97 \%)$ as a yellow solid $\left(\mathrm{mp}=138-141^{\circ} \mathrm{C}\right)$. ${ }^{1} \mathrm{H}$ NMR (400 MHz, $d_{6}$-DMSO) $\delta(\mathrm{ppm}) 9.98(\mathrm{~s}, 1 \mathrm{H}), 8.66(\mathrm{~d}, \mathrm{~J}=4.4 \mathrm{~Hz}$, $1 \mathrm{H}, \mathrm{H} 6), 8.43(\mathrm{~s}, 1 \mathrm{H}, \mathrm{H} 2), 8.37(\mathrm{~d}, \mathrm{~J}=8.2 \mathrm{~Hz}, 2 \mathrm{H}), 8.22(\mathrm{~d}, \mathrm{~J}=9.2 \mathrm{~Hz}$, $1 \mathrm{H}, \mathrm{H} 8), 7.96(\mathrm{~d}, \mathrm{~J}=8.2 \mathrm{~Hz}, 2 \mathrm{H}$ ), 7.33 (dd, $\mathrm{J}=4.4$ and $9.2 \mathrm{~Hz}, 1 \mathrm{H}, \mathrm{H} 7$ ). ${ }^{13} \mathrm{C}$ NMR (100 MHz, $d_{6}$-DMSO) $\delta(p p m)$ 192.6, 144.5, 141.2, 125.1, 135.0, 134.4, 130.2, 126.6, 126.4, 126.3, 118.3. Elemental analysis: calcd (\%) for $\mathrm{C}_{13} \mathrm{H}_{9} \mathrm{~N}_{3} \mathrm{O}$ (223.24): C 69.95, H 4.06; found: C 70.12, H 4.21.

1-(4-(Imidazo[1,2-b]pyridazin-3-yl)phenyl)ethan-1-one (5): Following the general procedure using 4-bromoacetophenone $(0.199 \mathrm{~g}, 1 \mathrm{mmol})$ and imidazo[1,2-b]pyridazine $(0.131 \mathrm{~g}, 1.1 \mathrm{mmol})$, the residue was purified by flash chromatography on silica gel (pentane- $\mathrm{Et}_{2} \mathrm{O}, 65: 35$ ) to 
afford the desired compound $5(0.218 \mathrm{~g}, 92 \%)$ as a yellow solid $(\mathrm{mp}=$ $\left.136-138{ }^{\circ} \mathrm{C}\right) .{ }^{1} \mathrm{H}$ NMR $\left(400 \mathrm{MHz}, d_{6}\right.$-DMSO) $\delta$ (ppm) 8.71 (dd, $J=1.6$ and $4.4 \mathrm{~Hz}, 1 \mathrm{H}, \mathrm{H6}), 8.45(\mathrm{~s}, 1 \mathrm{H}, \mathrm{H} 2), 8.36(\mathrm{~d}, \mathrm{~J}=8.4 \mathrm{~Hz}, 2 \mathrm{H}), 8.26(\mathrm{dd}$, $J=1.6$ and $9.2 \mathrm{~Hz}, 1 \mathrm{H}, \mathrm{H} 8), 8.08(\mathrm{~d}, \mathrm{~J}=8.4 \mathrm{~Hz}, 2 \mathrm{H}), 7.36$ (dd, $\mathrm{J}=4.4$ and $9.2 \mathrm{~Hz}, 1 \mathrm{H}, \mathrm{H} 7), 2.62(\mathrm{~s}, 3 \mathrm{H}) .{ }^{13} \mathrm{C}$ NMR $\left(100 \mathrm{MHz}, d_{6}\right.$-DMSO) $\delta$ (ppm) 197.2, 144.2, 140.6, 135.4, 134.3, 132.8, 128.7, 126.3, 126.2, 125.7, 117.8, 26.7. Elemental analysis: calcd (\%) for $\mathrm{C}_{14} \mathrm{H}_{11} \mathrm{~N}_{3} \mathrm{O}$ (237.26): C 70.87, H 4.67; found: C 71.13, H 4.51.

Ethyl 4-(imidazo[1,2-b]pyridazin-3-yl)benzoate (6): Following the general procedure using ethyl 4-bromobenzoate $(0.229 \mathrm{~g}, 1 \mathrm{mmol})$ and imidazo[1,2-b]pyridazine $(0.131 \mathrm{~g}, 1.1 \mathrm{mmol})$, the residue was purified by flash chromatography on silica gel (pentane- $\mathrm{Et}_{2} \mathrm{O}, 65: 35$ ) to afford the desired compound $6(0.243 \mathrm{~g}, 91 \%)$ as a pale yellow solid $(\mathrm{mp}=108-110$ $\left.{ }^{\circ} \mathrm{C}\right) .{ }^{1} \mathrm{H}$ NMR $\left(400 \mathrm{MHz}, d_{6}\right.$-DMSO) $\delta(\mathrm{ppm}) 8.75$ (dd, $J=1.6$ and $4.5 \mathrm{~Hz}$, $1 \mathrm{H}, \mathrm{H6}$ ), $8.49(\mathrm{~s}, 1 \mathrm{H}, \mathrm{H} 2), 8.35(\mathrm{~d}, \mathrm{~J}=8.5 \mathrm{~Hz}, 2 \mathrm{H}), 8.3$ (dd, $\mathrm{J}=1.9$ and $9.2 \mathrm{~Hz}, 1 \mathrm{H}, \mathrm{H} 8), 8.09(\mathrm{~d}, \mathrm{~J}=8.5 \mathrm{~Hz}, 2 \mathrm{H}), 7.42(\mathrm{dd}, \mathrm{J}=4.5$ and $9.2 \mathrm{~Hz}$, $1 \mathrm{H}, \mathrm{H} 7), 4.35$ (q, J = 7.1 Hz, 2H), $1.35(\mathrm{t}, J=7.1 \mathrm{~Hz}, 3 \mathrm{H}) .{ }^{13} \mathrm{C}$ NMR $(100$ $\mathrm{MHz}, d_{6}$-DMSO) $\delta$ (ppm) 165.4, 144.5, 140.2, 133.2, 132.6, 129.5, 128.6, 126.3, 126.0, 125.9, 118.5, 60.8, 14.2. Elemental analysis: calcd (\%) for $\mathrm{C}_{15} \mathrm{H}_{13} \mathrm{~N}_{3} \mathrm{O}_{2}$ (267.29): C 67.40, H 4.90; found: C 67.58, H 5.12.

3-(4-Chlorophenyl)imidazo[1,2-b]pyridazine (7): Following the general procedure using 1-bromo-4-chlorobenzene $(0.191 \mathrm{~g}, 1 \mathrm{mmol})$ and imidazo[1,2-b]pyridazine $(0.131 \mathrm{~g}, 1.1 \mathrm{mmol})$, the residue was purified by flash chromatography on silica gel (pentane- $\mathrm{Et}_{2} \mathrm{O}, 65: 35$ ) to afford the desired compound $7(0.204 \mathrm{~g}, 89 \%)$ as a pale yellow solid $(\mathrm{mp}=144-148$ $\left.{ }^{\circ} \mathrm{C}\right) .{ }^{1} \mathrm{H}$ NMR $\left(400 \mathrm{MHz}, d_{6}\right.$-DMSO) $\delta(\mathrm{ppm}) 8.66(\mathrm{dd}, J=1.6$ and $4.5 \mathrm{~Hz}$, $1 \mathrm{H}, \mathrm{H} 6), 8.32(\mathrm{~s}, 1 \mathrm{H}, \mathrm{H} 2), 8.25-8.19(\mathrm{~m}, 3 \mathrm{H}), 7.59(\mathrm{~d}, \mathrm{~J}=8.5 \mathrm{~Hz}, 2 \mathrm{H})$ 7.32 (dd, $J=4.5$ and $9.2 \mathrm{~Hz}, 1 \mathrm{H}, \mathrm{H} 7) .{ }^{13} \mathrm{C}$ NMR $\left(100 \mathrm{MHz}, d_{6}\right.$-DMSO) $\delta$ (ppm) 144.0, 140.0, 133.2, 132.1, 128.7, 127.8, 127.3, 126.1, 126.1, 117.4. Elemental analysis: calcd (\%) for $\mathrm{C}_{12} \mathrm{H}_{8} \mathrm{ClN}_{3}$ (229.67): C 62.76, $\mathrm{H}$ 3.51; found: C $62.80, \mathrm{H} 3.72$.

3-(4-Fluorophenyl)imidazo[1,2-b]pyridazine (8): Following the general procedure using 1-bromo-4-fluorobenzene $(0.175 \mathrm{~g}, 1 \mathrm{mmol})$ and imidazo[1,2-b]pyridazine $(0.131 \mathrm{~g}, 1.1 \mathrm{mmol})$, the residue was purified by flash chromatography on silica gel (pentane- $\mathrm{Et}_{2} \mathrm{O}, 70: 30$ ) to afford the desired compound $8(0.200 \mathrm{~g}, 94 \%)$ as a pale yellow solid $(\mathrm{mp}=108-111$ $\left.{ }^{\circ} \mathrm{C}\right) .{ }^{1} \mathrm{H}$ NMR $\left(400 \mathrm{MHz}, d_{6}\right.$-DMSO) $\delta(\mathrm{ppm}) 8.64(\mathrm{dd}, J=1.6$ and $4.4 \mathrm{~Hz}$, $1 \mathrm{H}, \mathrm{H} 6), 8.25(\mathrm{~s}, 1 \mathrm{H}, \mathrm{H} 2), 8.23-8.15(\mathrm{~m}, 3 \mathrm{H}), 7.36(\mathrm{t}, \mathrm{J}=8.9 \mathrm{~Hz}, 2 \mathrm{H})$, 7.29 (dd, $J=4.4$ and $9.2 \mathrm{~Hz}, 1 \mathrm{H}, \mathrm{H} 7) .{ }^{13} \mathrm{C}$ NMR $\left(100 \mathrm{MHz}, d_{6}\right.$-DMSO) $\delta$ (ppm) $161.5(\mathrm{~d}, J=244.5 \mathrm{~Hz}), 143.9,139.7,132.7,128.4(\mathrm{~d}, J=8.2 \mathrm{~Hz})$, 126.4, 126.1, $125.0(\mathrm{~d}, \mathrm{~J}=3.0 \mathrm{~Hz}), 117.1,115.6(\mathrm{~d}, \mathrm{~J}=19.4 \mathrm{~Hz})$. Elemental analysis: calcd (\%) for $\mathrm{C}_{12} \mathrm{H}_{8} \mathrm{FN}_{3}$ (213.22): C 67.60, H 3.78; found: C $67.69, \mathrm{H} 3.66$.

3-(P-tolyl)imidazo[1,2-b]pyridazine (9): Following the general procedure using 4-bromotoluene $(0.171 \mathrm{~g}, 1 \mathrm{mmol})$ and imidazo[1,2b]pyridazine $(0.131 \mathrm{~g}, 1.1 \mathrm{mmol})$, the residue was purified by flash chromatography on silica gel (pentane- $\mathrm{Et}_{2} \mathrm{O}, 70: 30$ ) to afford the desired compound $9(0.172 \mathrm{~g}, 82 \%)$ as a yellow solid $\left(\mathrm{mp}=105-108{ }^{\circ} \mathrm{C}\right) .{ }^{1} \mathrm{H}$ $\operatorname{NMR}\left(400 \mathrm{MHz}, d_{6}\right.$-DMSO) $\delta(\mathrm{ppm}) 8.63(\mathrm{dd}, J=1.6$ and $4.5 \mathrm{~Hz}, 1 \mathrm{H}, \mathrm{H} 6)$ $8.22(\mathrm{~s}, 1 \mathrm{H}, \mathrm{H} 2), 8.20(\mathrm{~d}, \mathrm{~J}=9.2 \mathrm{~Hz}, 1 \mathrm{H}, \mathrm{H} 8), 8.04(\mathrm{~d}, \mathrm{~J}=8.2 \mathrm{~Hz}, 2 \mathrm{H})$, $7.33(\mathrm{~d}, \mathrm{~J}=8.2 \mathrm{~Hz}, 2 \mathrm{H}), 7.27$ (dd, $\mathrm{J}=4.7$ and $9.2 \mathrm{~Hz}, 1 \mathrm{H}, \mathrm{H} 7$ ), 2.36 (s, $3 \mathrm{H}) .{ }^{13} \mathrm{C}$ NMR $\left(100 \mathrm{MHz}, d_{6}\right.$-DMSO) $\delta(\mathrm{ppm}) 143.8,139.6,137.2,132.4$, 129.2, 127.3, 126.2, 126.0, 125.6, 116.8, 20.9. Elemental analysis: calcd (\%) for $\mathrm{C}_{13} \mathrm{H}_{11} \mathrm{~N}_{3}$ (209.25) C 74.62, H 5.30; found: C 74.97, H 5.46.

4-(Imidazo[1,2-b]pyridazin-3-yl)aniline (10): Following the general procedure using 4-iodoaniline $(0.219 \mathrm{~g}, 1 \mathrm{mmol})$ and imidazo[1,2b]pyridazine $(0.131 \mathrm{~g}, 1.1 \mathrm{mmol})$, the residue was purified by flash chromatography on silica gel $\left(\mathrm{CH}_{2} \mathrm{Cl}_{2}-\mathrm{MeOH}, 98: 2\right)$ to afford the desired compound $10(0.191 \mathrm{~g}, 91 \%)$ as a yellow solid $\left(\mathrm{mp}=150-152{ }^{\circ} \mathrm{C}\right) .{ }^{1} \mathrm{H}$ NMR (400 MHz, $d_{6}$-DMSO) $\delta(\mathrm{ppm}) 8.57$ (dd, $J=1.6$ and $4.5 \mathrm{~Hz}, 1 \mathrm{H}, \mathrm{H} 6$ ),
8.13 (dd, $\mathrm{J}=1.9$ and $9.2 \mathrm{~Hz}, 1 \mathrm{H}, \mathrm{H} 8$ ), $8.11(\mathrm{~s}, 1 \mathrm{H}, \mathrm{H} 2), 7.80$ (d, $\mathrm{J}=8.5$ $\mathrm{Hz}, 2 \mathrm{H}), 7.18(\mathrm{dd}, J=4.7$ and $9.2 \mathrm{~Hz}, 1 \mathrm{H}, \mathrm{H7}), 6.69(\mathrm{~d}, J=8.5 \mathrm{~Hz}, 2 \mathrm{H})$, 5.37 (brs, $2 \mathrm{H}$ ). ${ }^{13} \mathrm{C}$ NMR (100 MHz, $d_{6}$-DMSO) $\delta$ (ppm) 148.7, 143.4, $138.8,130.8,128.4,127.5,115.8,115.7,113.6$. Elemental analysis: calcd (\%) for $\mathrm{C}_{12} \mathrm{H}_{10} \mathrm{~N}_{4}(210.24)$ C 68.56, H 4.79; found: C 68.84, H 4.56.

Methyl 3-(imidazo[1,2-b]pyridazin-3-yl)benzoate (11): Following the general procedure using methyl 3-bromobenzoate $(0.215 \mathrm{~g}, 1 \mathrm{mmol})$ and imidazo[1,2-b]pyridazine $(0.131 \mathrm{~g}, 1.1 \mathrm{mmol})$, the residue was purified by flash chromatography on silica gel (pentane- $\mathrm{Et}_{2} \mathrm{O}, 70: 30$ ) to afford the desired compound $11(0.233 \mathrm{~g}, 92 \%)$ as a pale yellow solid $(\mathrm{mp}=119$ $\left.121^{\circ} \mathrm{C}\right) .{ }^{1} \mathrm{H}$ NMR $\left(400 \mathrm{MHz}, d_{6}\right.$-DMSO) $\delta(\mathrm{ppm}) 8.78(\mathrm{~s}, 1 \mathrm{H}), 8.69$ (dd, $J$ $=1.7$ and $4.6 \mathrm{~Hz}, 1 \mathrm{H}, \mathrm{H} 6), 8.38(\mathrm{~d}, J=7.8 \mathrm{~Hz}, 1 \mathrm{H}), 8.36(\mathrm{~s}, 1 \mathrm{H}, \mathrm{H} 2), 8.24$ (dd, $J=1.7$ and $9.2 \mathrm{~Hz}, 1 \mathrm{H}, \mathrm{H} 8$ ), 7.96 (ddd, $J=6.1,6.4$ and $7.8 \mathrm{~Hz}, 1 \mathrm{H}$ ), $7.67(\mathrm{dd}, J=7.5$ and $7.8 \mathrm{~Hz}, 1 \mathrm{H}), 7.33(\mathrm{dd}, J=4.4$ and $9.2 \mathrm{~Hz}, 1 \mathrm{H}, \mathrm{H} 7$ ), 3.90 (s, 3H). ${ }^{13} \mathrm{C}$ NMR (100 MHz, $d_{6}$-DMSO) $\delta$ (ppm) 166.1, 144.1, 140.1, 133.4, 130.9, 130.1, 129.2, 129.0, 128.3, 126.5, 126.2, 117.5, 52.3 Elemental analysis: calcd (\%) for $\mathrm{C}_{14} \mathrm{H}_{11} \mathrm{~N}_{3} \mathrm{O}_{2}(253.26) \mathrm{C} 66.40, \mathrm{H}$ 4.38; found: C $66.57, \mathrm{H} 4.71$.

1-(3-(Imidazo[1,2-b]pyridazin-3-yl)phenyl)ethan-1-one (12): Following the general procedure using 3-bromoacetophenone $(0.197 \mathrm{~g}, 1 \mathrm{mmol})$ and imidazo[1,2-b]pyridazine $(0.131 \mathrm{~g}, 1.1 \mathrm{mmol})$, the residue was purified by flash chromatography on silica gel $\left(\mathrm{CH}_{2} \mathrm{Cl}_{2}-\mathrm{MeOH}, 98: 2\right)$ to afford the desired compound $12(0.211 \mathrm{~g}, 89 \%)$ as a pale yellow solid $\left(\mathrm{mp}=143-146^{\circ} \mathrm{C}\right) .{ }^{1} \mathrm{H} \mathrm{NMR}\left(400 \mathrm{MHz}, d_{6}\right.$-DMSO) $\delta(\mathrm{ppm}) 8.72(\mathrm{~s}, 1 \mathrm{H})$, $8.69(\mathrm{dd}, \mathrm{J}=1.6$ and $4.5 \mathrm{~Hz}, 1 \mathrm{H}, \mathrm{H} 6), 8.39(\mathrm{~d}, \mathrm{~J}=7.7 \mathrm{~Hz}, 1 \mathrm{H}), 8.39(\mathrm{~s}$, $1 \mathrm{H}, \mathrm{H} 2$ ), 8.25 (dd, $\mathrm{J}=1.6$ and $9.2 \mathrm{~Hz}, 1 \mathrm{H}, \mathrm{H} 8$ ), 7.98 (ddd, $J=6.5,6.7$ and $7.7 \mathrm{~Hz}, 1 \mathrm{H}$ ), 7.68 (dd, $J=7.1$ and $7.4 \mathrm{~Hz}, 1 \mathrm{H}$ ), 7.34 (dd, $J=4.4$ and $9.2 \mathrm{~Hz}, 1 \mathrm{H}, \mathrm{H} 7), 2.67(\mathrm{~s}, 3 \mathrm{H}) .{ }^{13} \mathrm{C} \mathrm{NMR}\left(100 \mathrm{MHz}, d_{6}-\mathrm{DMSO}\right) \delta(\mathrm{ppm})$ $197.8,144.1,140.0,137.2,133.4,130.6,129.1,128.9,127.4,126.4$, 126.2, 125.7, 117.5, 26.9. Elemental analysis: calcd (\%) for $\mathrm{C}_{14} \mathrm{H}_{11} \mathrm{~N}_{3} \mathrm{O}$ (237.26) C 70.87, H 4.67; found: C 71.05, H 4.98 .

3-(3-Bromophenyl)imidazo[1,2-b]pyridazine (13): Following the general procedure using 1,3-dibromobenzene $(0.236 \mathrm{~g}, 1 \mathrm{mmol})$ and imidazo[1,2-b]pyridazine $(0.131 \mathrm{~g}, 1.1 \mathrm{mmol})$, the residue was purified by flash chromatography on silica gel (pentane- $\mathrm{Et}_{2} \mathrm{O}, 70: 30$ ) to afford the desired compound $13(0.233 \mathrm{~g}, 85 \%)$ as a grey solid $\left(\mathrm{mp}=92-95^{\circ} \mathrm{C}\right)$. ${ }^{1} \mathrm{H}$ NMR $\left(400 \mathrm{MHz}, d_{6}\right.$-DMSO) $\delta(\mathrm{ppm}) 8.69$ (dd, $J=1.7$ and $4.5 \mathrm{~Hz}, 1 \mathrm{H}$, $\mathrm{H} 6), 8.44(\mathrm{t}, \mathrm{J}=1.9 \mathrm{~Hz}, 1 \mathrm{H}), 8,38(\mathrm{~s}, 1 \mathrm{H}, \mathrm{H} 2), 8.24(\mathrm{dd}, \mathrm{J}=1.7$ and 9.1 $\mathrm{Hz}, 1 \mathrm{H}, \mathrm{H} 8$ ), 8.17 (ddd, $J=6.1,6.7$ and $7.8 \mathrm{~Hz}, 1 \mathrm{H}$ ), 7.57 (d, $J=7.8 \mathrm{~Hz}$, $1 \mathrm{H}$ ), 7.48 (dd, $J=7.1$ and $7.4 \mathrm{~Hz}, 1 \mathrm{H}$ ), 7.33 (dd, $J=4.5$ and $9.1 \mathrm{~Hz}, 1 \mathrm{H}$ H7). ${ }^{13} \mathrm{C}$ NMR $\left(100 \mathrm{MHz}, d_{6}\right.$-DMSO) $\delta(\mathrm{ppm}) 144.1,140.2,133.6,130.8$, 130.7, 130.3, 128.2, 126.2, 125.6, 125.0, 122.0, 117.6. Elemental analysis: calcd (\%) for $\mathrm{C}_{12} \mathrm{H}_{8} \mathrm{BrN}_{3}$ (274.12) C 52.58, H 2.94; found: C $52.67, \mathrm{H} 3.14$.

3-(Imidazo[1,2-b]pyridazin-3-yl)aniline (14): Following the general procedure using 3-iodoaniline $(0.219 \mathrm{~g}, 1 \mathrm{mmol})$ and imidazo[1,2b]pyridazine $(0.131 \mathrm{~g}, 1.1 \mathrm{mmol})$, the residue was purified by flash chromatography on silica gel $\left(\mathrm{CH}_{2} \mathrm{Cl}_{2}-\mathrm{MeOH}, 98: 2\right)$ to afford the desired compound $14(0.172 \mathrm{~g}, 82 \%)$ as a brown solid $\left(\mathrm{mp}=112-115^{\circ} \mathrm{C}\right) .{ }^{1} \mathrm{H}$ NMR (400 MHz, $d_{6}$-DMSO) $\delta(\mathrm{ppm}) 8.61(\mathrm{dd}, J=1.6$ and $4.5 \mathrm{~Hz}, 1 \mathrm{H}, \mathrm{H6}$ ), 8.18 (dd, $J=1.6$ and $9.2 \mathrm{~Hz}, 1 \mathrm{H}, \mathrm{H} 8$ ), $8.08(\mathrm{~s}, 1 \mathrm{H}, \mathrm{H} 2), 7.35$ (dd, $\mathrm{J}=2.0$ and $2.1 \mathrm{~Hz}, 1 \mathrm{H}), 7.29-7.20(\mathrm{~m}, 2 \mathrm{H}), 7.14(\mathrm{dd}, J=7.5$ and $7.6 \mathrm{~Hz}, 1 \mathrm{H}), 6.6$ (md, $J=7.9 \mathrm{~Hz}, 1 \mathrm{H}), 5.20$ (brs, $2 \mathrm{H}) .{ }^{13} \mathrm{C}$ NMR $\left(100 \mathrm{MHz}, d_{6}\right.$-DMSO) $\delta$ (ppm) 148.8, 143.7, 139.5, 132.4, 129.1, 128.8, 128.1, 125.9, 116.7, 114.3, 113.6, 111.7. Elemental analysis: calcd (\%) for $\mathrm{C}_{12} \mathrm{H}_{10} \mathrm{~N}_{4}(210.24)$ C 68.56, H 4.79; found: C 68.79, H 4.89 .

3-(3,5-Bis(trifluoromethyl)phenyl)imidazo[1,2-b]pyridazine (15): Following the general procedure using $3,5-$ bis(trifluoromethyl)bromobenzene $(0.293 \mathrm{~g}, 1 \mathrm{mmol})$ and imidazo[1,2b]pyridazine $(0.131 \mathrm{~g}, 1.1 \mathrm{mmol})$, the residue was purified by flash 
chromatography on silica gel (pentane- $\mathrm{Et}_{2} \mathrm{O}, 70: 30$ ) to afford the desired compound $15(0.298 \mathrm{~g}, 90 \%)$ as a grey solid $\left(\mathrm{mp}=156-159{ }^{\circ} \mathrm{C}\right) .{ }^{1} \mathrm{H}$ NMR (400 MHz, $d_{6}$-DMSO) $\delta(\mathrm{ppm}) 8.88(\mathrm{~s}, 2 \mathrm{H}), 8.77(\mathrm{dd}, J=1.6$ and $4.5 \mathrm{~Hz}, 1 \mathrm{H}, \mathrm{H} 6$ ), 8.66 (s, $1 \mathrm{H}$ ), 8.29 (dd, $J=1.6$ and $9.2 \mathrm{~Hz}, 1 \mathrm{H}, \mathrm{H} 8$ ), 8.06 (s, $1 \mathrm{H}, \mathrm{H} 2), 7.4$ (dd, $J=4.5$ and $9.2 \mathrm{~Hz}, 1 \mathrm{H}, \mathrm{H} 7) .{ }^{13} \mathrm{C} \mathrm{NMR}\left(100 \mathrm{MHz}, d_{6^{-}}\right.$ DMSO) $\delta$ (ppm) 141.2, 140.6, 134.8, 130.9, 128.4 (q, $J=44.9 \mathrm{~Hz}), 125.6$ 124.6, 124.0, 123.1 (q, $J=272.1 \mathrm{~Hz}$ ), 120.2, 118.1. Elemental analysis: calcd (\%) for $\mathrm{C}_{14} \mathrm{H}_{7} \mathrm{~F}_{6} \mathrm{~N}_{3}$ (331.22) C 50.77, H 2.13; found: C 50.98, H 2.08 .

2-(Imidazo[1,2-b]pyridazin-3-yl)benzonitrile (16): Following the general procedure using 2-bromobenzonitrile $(0.182 \mathrm{~g}, 1 \mathrm{mmol})$ and imidazo[1,2-b]pyridazine $(0.131 \mathrm{~g}, 1.1 \mathrm{mmol})$, the residue was purified by flash chromatography on silica gel (pentane- $\mathrm{Et}_{2} \mathrm{O}, 70: 30$ ) to afford the desired compound $16(0.205 \mathrm{~g}, 93 \%)$ as a grey solid $\left(\mathrm{mp}=214-218^{\circ} \mathrm{C}\right)$. ${ }^{1} \mathrm{H}$ NMR $\left(400 \mathrm{MHz}, d_{6}\right.$-DMSO) $\delta(\mathrm{ppm}) 8.62(\mathrm{dd}, J=1.6$ and $4.5 \mathrm{~Hz}, 1 \mathrm{H}$, $\mathrm{H} 6$ ), 8.28 (dd, $\mathrm{J}=1.6$ and $9.2 \mathrm{~Hz}, 1 \mathrm{H}, \mathrm{H} 8), 8.18(\mathrm{~s}, 1 \mathrm{H}, \mathrm{H} 2), 8.04(\mathrm{~d}, \mathrm{~J}=$ $7.6 \mathrm{~Hz}, 1 \mathrm{H}), 7.99(\mathrm{~d}, J=7.8 \mathrm{~Hz}, 1 \mathrm{H}), 7.88(\mathrm{dd}, J=7.6$ and $7.8 \mathrm{~Hz}, 1 \mathrm{H})$, $7.66(\mathrm{~d}, \mathrm{~J}=7.6$ and $7.8 \mathrm{~Hz}, 1 \mathrm{H}), 7.38$ (dd, $\mathrm{J}=4.4$ and $9.2 \mathrm{~Hz}, 1 \mathrm{H}, \mathrm{H} 7$ ). ${ }^{13} \mathrm{C}$ NMR (100 MHz, $d_{6}$-DMSO) $\delta(\mathrm{ppm}) 144.0,139.7,134.3,133.7$, 133.3, 131.1, 130.5, 129.1, 126.2, 124.4, 118.5, 118.1, 111.3. Elemental analysis: calcd (\%) for $\mathrm{C}_{13} \mathrm{H}_{8} \mathrm{~N}_{4}$ (220.23): C 70.90, H 3.66; found: C 71.03 H 3.52 .

2-(Imidazo[1,2-b]pyridazin-3-yl)benzaldehyde (17): Following the general procedure using 2-bromobenzaldehyde $(0.183 \mathrm{~g}, 1 \mathrm{mmol})$ and imidazo[1,2-b]pyridazine $(0.131 \mathrm{~g}, 1.1 \mathrm{mmol})$, the residue was purified by flash chromatography on silica gel (pentane- $\mathrm{Et}_{2} \mathrm{O}, 65: 35$ ) to afford the desired compound $17(0.203 \mathrm{~g}, 91 \%)$ as a grey solid $\left(\mathrm{mp}=157-160{ }^{\circ} \mathrm{C}\right)$. ${ }^{1} \mathrm{H}$ NMR $\left(400 \mathrm{MHz}, d_{6}-\mathrm{DMSO}\right) \delta(\mathrm{ppm}) 9.76(\mathrm{~s}, 1 \mathrm{H}), 8.51$ (dd, $\mathrm{J}=1.6$ and $4.4 \mathrm{~Hz}, 1 \mathrm{H}, \mathrm{H} 6), 8.24$ (dd, $\mathrm{J}=1.7$ and $9.3 \mathrm{~Hz}, 1 \mathrm{H}, \mathrm{H} 8$ ), $8.08(\mathrm{~s}, 1 \mathrm{H}, \mathrm{H} 2$ ), 8.02 (dd, $J=1.5$ and $7.7 \mathrm{~Hz}, 1 \mathrm{H}$ ), $7.83(\mathrm{dt}, \mathrm{J}=1.5$ and $7.6 \mathrm{~Hz}, 1 \mathrm{H}$ ), $7.75-$ $7.66(\mathrm{~m}, 2 \mathrm{H}), 7.32(\mathrm{dd}, J=4.5$ and $9.2 \mathrm{~Hz}, 1 \mathrm{H}, \mathrm{H} 7) .{ }^{13} \mathrm{C} \mathrm{NMR}(100 \mathrm{MHz}$, $d_{6}$-DMSO) $\delta(\mathrm{ppm}) 191.4,144.0,139.5,134.5,134.1,133.9,131.7$, $130.0,129.2,128.3,126.1,125.2,118.1$. Elemental analysis: calcd (\%) for $\mathrm{C}_{13} \mathrm{H}_{9} \mathrm{~N}_{3} \mathrm{O}$ (223.24): C 69.95, $\mathrm{H} 4.06$; found: $\mathrm{C} 69.79, \mathrm{H} 4.41$.

3-(2-(Trifluoromethyl)phenyl)imidazo[1,2-b]pyridazine (18): Following the general procedure using 2-(trifluoromethyl)bromobenzene $(0.225 \mathrm{~g}, 1$ $\mathrm{mmol}$ ) and imidazo[1,2-b]pyridazine $(0.131 \mathrm{~g}, 1.1 \mathrm{mmol})$, the residue was purified by flash chromatography on silica gel (pentane- $\left.\mathrm{Et}_{2} \mathrm{O}, 65: 35\right)$ to afford the desired compound $18(0.253 \mathrm{~g}, 96 \%)$ as a yellow solid $(\mathrm{mp}=$ 176-179 $\left.{ }^{\circ} \mathrm{C}\right) .{ }^{1} \mathrm{H}$ NMR $\left(400 \mathrm{MHz}, d_{6}\right.$-DMSO) $\delta(\mathrm{ppm}) 8.49$ (dd, $\mathrm{J}=1.5$ and $4.5 \mathrm{~Hz}, 1 \mathrm{H}, \mathrm{H6}$ ), $8.22(\mathrm{dd}, J=1.6$ and $9.2 \mathrm{~Hz}, 1 \mathrm{H}, \mathrm{H} 8), 7.94(\mathrm{~d}, J=$ $7.7 \mathrm{~Hz}, 1 \mathrm{H}), 7.86(\mathrm{~s}, 1 \mathrm{H}), 7.82(\mathrm{dd}, J=6.9$ and $8.7 \mathrm{~Hz}, 1 \mathrm{H}), 7.74(\mathrm{dd}, J=$ 6.9 and $7.7 \mathrm{~Hz}, 1 \mathrm{H}), 7.69(\mathrm{~d}, J=7.7 \mathrm{~Hz}, 1 \mathrm{H}), 7.3(\mathrm{dd}, J=4.5$ and $9.2 \mathrm{~Hz}$, $1 \mathrm{H}, \mathrm{H} 7) .{ }^{13} \mathrm{C}$ NMR $\left(100 \mathrm{MHz}, d_{6}\right.$-DMSO) $\delta(\mathrm{ppm}) 143.8,138.7,133.7$, $133.5,132.4,129.8,120.0(q, J=29.1 \mathrm{~Hz}), 126.6,126.4(q, J=2.1 \mathrm{~Hz})$, $125.8,124.5,123.8(\mathrm{q}, J=274.2 \mathrm{~Hz}), 117.8$. Elemental analysis: calcd (\%) for $\mathrm{C}_{13} \mathrm{H}_{8} \mathrm{~F}_{3} \mathrm{~N}_{3}$ (263.22): C 59.32, H 3.06; found: C 59.51, H 2.85.

3-(Naphthalen-1-yl)imidazo[1,2-b]pyridazine (19): Following the general procedure using 1 -bromonaphthalene $(0.207 \mathrm{~g}, 1 \mathrm{mmol})$ and imidazo[1,2-b]pyridazine $(0.131 \mathrm{~g}, 1.1 \mathrm{mmol})$, the residue was purified by flash chromatography on silica gel (pentane- $\mathrm{Et}_{2} \mathrm{O}, 65: 35$ ) to afford the desired compound $19(0.182 \mathrm{~g}, 74 \%)$ as a yellow solid $(\mathrm{mp}=173-176$ $\left.{ }^{\circ} \mathrm{C}\right) .{ }^{1} \mathrm{H}$ NMR $\left(400 \mathrm{MHz}, d_{6}\right.$-DMSO) $\delta(\mathrm{ppm}) 8.46(\mathrm{dd}, J=1.7$ and $4.4 \mathrm{~Hz}$, $1 \mathrm{H}, \mathrm{H} 6), 8.27(\mathrm{dd}, \mathrm{J}=1.7$ and $9.2 \mathrm{~Hz}, 1 \mathrm{H}, \mathrm{H} 8), 8.11-8.02(\mathrm{~m}, 3 \mathrm{H}), 7.74$ (dd, $J=1.6$ and $7.1 \mathrm{~Hz}, 1 \mathrm{H}$ ), 7.66 (dd, $J=7.1$ and $7.4 \mathrm{~Hz}, 1 \mathrm{H}$ ), 6.60-7.51 (m, $2 \mathrm{H}), 7.50-7.44(\mathrm{~m}, 1 \mathrm{H}), 7.30(\mathrm{dd}, \mathrm{J}=4.4$ and $9.2 \mathrm{~Hz}, 1 \mathrm{H}, \mathrm{H} 7) .{ }^{13} \mathrm{C}$ NMR $\left(100 \mathrm{MHz}, d_{6}\right.$-DMSO) $\delta(\mathrm{ppm}) 143.7,139.0,134.0,133.3,131.4$, 129.2, 128.9, 128.4, 126.7, 126.6, 126.2, 125.9, 125.7, 125.4, 125.3, 117.5. Elemental analysis: calcd (\%) for $\mathrm{C}_{16} \mathrm{H}_{11} \mathrm{~N}_{3}$ (245.28): C 78.35, $\mathrm{H}$ 4.52; found: C 78.57, H 4.59 .
3-(Pyren-1-yl)imidazo[1,2-b]pyridazine (20): Following the general procedure using 1 -bromopyrene $(0.281 \mathrm{~g}, 1 \mathrm{mmol})$ and imidazo[1,2b]pyridazine $(0.131 \mathrm{~g}, 1.1 \mathrm{mmol})$, the residue was purified by flash chromatography on silica gel (pentane- $\mathrm{Et}_{2} \mathrm{O}, 80: 20$ ) to afford the desired compound $20(0.252 \mathrm{~g}, 79 \%)$ as a yellow solid $\left(\mathrm{mp}=190-194{ }^{\circ} \mathrm{C}\right) .{ }^{1} \mathrm{H}$ NMR (400 MHz, $d_{6}$-DMSO) $\delta(\mathrm{ppm}) 8.50$ (dd, $\mathrm{J}=1.6$ and $\left.4.6 \mathrm{~Hz}, 1 \mathrm{H}, \mathrm{H} 6\right)$, $8.42(\mathrm{~d}, \mathrm{~J}=8.2 \mathrm{~Hz}, 1 \mathrm{H}), 8.37(\mathrm{~d}, \mathrm{~J}=7.8 \mathrm{~Hz}, 1 \mathrm{H}), 8.34-8.30(\mathrm{~m}, 2 \mathrm{H})$, 8.29-8.25 (m, 3H), $8.20(\mathrm{~s}, 1 \mathrm{H}, \mathrm{H} 2), 8.16(\mathrm{~d}, \mathrm{~J}=9.2 \mathrm{~Hz}, 1 \mathrm{H}), 8.12$ (dd, $\mathrm{J}$ $=7.4$ and $7.6 \mathrm{~Hz}, 1 \mathrm{H}), 7.89(\mathrm{dd}, J=1.6$ and $9.2 \mathrm{~Hz}, 1 \mathrm{H}, \mathrm{H} 8), 7.34(\mathrm{dd}, J$ $=4.4$ and $9.2 \mathrm{~Hz}, 1 \mathrm{H}, \mathrm{H} 7) .{ }^{13} \mathrm{C}$ NMR $\left(100 \mathrm{MHz}, d_{6}\right.$-DMSO) $\delta$ (ppm) 123.7, $124.0,124.7,124.8,125.4,125.7,126.0,126.5,126.8,127.3,128.1$, $128.7,129.2,130.3,130.8,131.1,134.6,139.2,143.9,117.7,122.9$ Elemental analysis: calcd (\%) for $\mathrm{C}_{22} \mathrm{H}_{13} \mathrm{~N}_{3}$ (319.36): C 82.74, H 4.10; found: C $82.89, \mathrm{H} 3.87$.

3-(Imidazo[1,2-b]pyridazin-3-yl)quinoline (21): Following the general procedure using 3-bromoquinoline $(0.208 \mathrm{~g}, 1 \mathrm{mmol})$ and imidazo[1,2 b]pyridazine $(0.131 \mathrm{~g}, 1.1 \mathrm{mmol})$, the residue was purified by flash chromatography on silica gel (pentane- $\mathrm{Et}_{2} \mathrm{O}, 55: 45$ ) to afford the desired compound $21(0.214 \mathrm{~g}, 87 \%)$ as a yellow solid $\left(\mathrm{mp}=230-233^{\circ} \mathrm{C}\right) .{ }^{1} \mathrm{H}$ NMR (400 MHz, $d_{6}$-DMSO) $\delta(p p m) 9.58(\mathrm{~d}, \mathrm{~J}=2.3 \mathrm{~Hz}, 1 \mathrm{H}), 9.20(\mathrm{~d}, \mathrm{~J}=$ $2.4 \mathrm{~Hz}, 1 \mathrm{H}$ ), $8.76(\mathrm{dd}, J=1.7$ and $4.4 \mathrm{~Hz}, 1 \mathrm{H}, \mathrm{H6}$ ), $8.58(\mathrm{~s}, 1 \mathrm{H}, \mathrm{H} 2), 8.29$ (dd, $J=1.7$ and $9.2 \mathrm{~Hz}, 1 \mathrm{H}, \mathrm{H} 8$ ), $8.07(\mathrm{dd}, J=3.1$ and $8.2 \mathrm{~Hz}, 2 \mathrm{H}$ ), 7.78 (ddd, $J=1.4,6.8$, and $8.5 \mathrm{~Hz}, 1 \mathrm{H}$ ), $7.66(\mathrm{dd}, J=7.0$ and $8.2 \mathrm{~Hz}, 1 \mathrm{H}$ ), 7.38 (dd, $J=4.5$ and $9.2 \mathrm{~Hz}, 1 \mathrm{H}, \mathrm{H} 7) .{ }^{13} \mathrm{C}$ NMR $\left(100 \mathrm{MHz}, d_{6}\right.$-DMSO) $\delta$ (ppm) 148.8, 146.4, 144.3, 140.4, 133.7, 131.2, 129.6, 128.8, 128.4, 127.3, 127.3, 126.2, 124.7, 122.0, 117.9. Elemental analysis: calcd $(\%)$ for $\mathrm{C}_{15} \mathrm{H}_{10} \mathrm{~N}_{4}$ (246.27): C 73.16, H 4.09; found: C 73.27, H 4.19.

3-(Pyridin-3-yl)imidazo[1,2-b]pyridazine (22): Following the genera procedure using 3-bromopyridine $(0.157 \mathrm{~g}, 1 \mathrm{mmol})$ and imidazo[1,2b]pyridazine $(0.131 \mathrm{~g}, 1.1 \mathrm{mmol})$, the residue was purified by flash chromatography on silica gel (pentane- $\mathrm{Et}_{2} \mathrm{O}, 55: 45$ ) to afford the desired compound $22(0.184 \mathrm{~g}, 94 \%)$ as a grey solid $\left(\mathrm{mp}=118-120{ }^{\circ} \mathrm{C}\right) .{ }^{1} \mathrm{H}$ NMR (400 MHz, $d_{6}$-DMSO) $\delta(\mathrm{ppm}) 9.31(\mathrm{~d}, \mathrm{~J}=2.3 \mathrm{~Hz}, 1 \mathrm{H}), 8.67$ (d, $\mathrm{J}=$ $4.5 \mathrm{~Hz}, 1 \mathrm{H}, \mathrm{H} 6), 8.57(\mathrm{~d}, J=5.3 \mathrm{~Hz}, 1 \mathrm{H}), 8.54$ (ddd, $J=2.1,7.5$ and 7.7 $\mathrm{Hz}, 1 \mathrm{H}$ ), 8.38 (s, 1H, H2), 8.25 (d, J = 9.2 Hz, 1H, H8), 7.54 (dd, J = 5.1 and $7.7 \mathrm{~Hz}, 1 \mathrm{H}), 7.33(\mathrm{dd}, J=4.4$ and $9.2 \mathrm{~Hz}, 1 \mathrm{H}, \mathrm{H} 7) .{ }^{13} \mathrm{C}$ NMR $(100$ $\mathrm{MHz}, d_{6}$-DMSO) $\delta$ (ppm) 148.4, 147.1, 144.1, 140.2, 133.2, 133.2, 126.2, 124.7, 124.5, 123.6, 117.7. Elemental analysis: calcd (\%) for $\mathrm{C}_{11} \mathrm{H}_{8} \mathrm{~N}_{4}$ (196.21): C 67.34, H 4.11; found: C 67.58, H 4.27.

3-(Pyrimidin-5-yl)imidazo[1,2-b]pyridazine (23): Following the general procedure using 5-bromopyrimidine $(0.159 \mathrm{~g}, 1 \mathrm{mmol})$ and imidazo[1,2b]pyridazine $(0.131 \mathrm{~g}, 1.1 \mathrm{mmol})$, the residue was purified by flash chromatography on silica gel (pentane- $\mathrm{Et}_{2} \mathrm{O}, 55: 45$ ) to afford the desired compound $23(0.144 \mathrm{~g}, 73 \%)$ as a grey solid $\left(\mathrm{mp}=230-234{ }^{\circ} \mathrm{C}\right) .{ }^{1} \mathrm{H}$ NMR (400 MHz, $d_{6}$-DMSO) $\delta(\mathrm{ppm}) 8.56(\mathrm{~s}, 2 \mathrm{H}), 9.18(\mathrm{~s}, 1 \mathrm{H}), 8.72(\mathrm{dd}, J$ $=1.6$ and $4.5 \mathrm{~Hz}, 1 \mathrm{H}, \mathrm{H} 6), 8.50(\mathrm{~s}, 1 \mathrm{H}, \mathrm{H} 2), 8.29(\mathrm{dd}, \mathrm{J}=1.6$ and $9.2 \mathrm{~Hz}$, $1 \mathrm{H}, \mathrm{H} 8), 7.39$ (dd, $\mathrm{J}=4.4$ and $9.2 \mathrm{~Hz}, 1 \mathrm{H}, \mathrm{H} 7) .{ }^{13} \mathrm{C} \mathrm{NMR}\left(100 \mathrm{MHz}, d_{6^{-}}\right.$ DMSO) $\delta$ (ppm) 171.4, 156.9, 153.6, 144.4, 140.6, 133.8, 126.3, 123.4, 118.3. Elemental analysis: calcd (\%) for $\mathrm{C}_{10} \mathrm{H}_{7} \mathrm{~N}_{5}$ (197.20): $\mathrm{C} 60.91, \mathrm{H}$ 3.58; found: C $61.17, \mathrm{H} 3.79$.

3-(4-(Trifluoromethyl)phenyl)imidazo[1,2-b]pyridazine (24): Following the general procedure using 4-(trifluoromethyl)chlorobenzene $(0.180 \mathrm{~g}, 1$ $\mathrm{mmol}$ ) and imidazo[1,2-b]pyridazine $(0.131 \mathrm{~g}, 1.1 \mathrm{mmol})$, the residue was purified by flash chromatography on silica gel (pentane- $\left.\mathrm{Et}_{2} \mathrm{O}, 65: 35\right)$ to afford the desired compound $24(0.182 \mathrm{~g}, 69 \%)$ as a yellow solid $(\mathrm{mp}=$ $\left.94-98{ }^{\circ} \mathrm{C}\right) .{ }^{1} \mathrm{H}$ NMR $\left(400 \mathrm{MHz}, d_{6}-\mathrm{DMSO}\right) \delta(\mathrm{ppm}) 8.70(\mathrm{dd}, J=1.6$ and $4.4 \mathrm{~Hz}, 1 \mathrm{H}, \mathrm{H} 6), 8.44$ (s, $1 \mathrm{H}, \mathrm{H} 2), 8.42(\mathrm{~d}, \mathrm{~J}=8.8 \mathrm{~Hz}, 2 \mathrm{H}$ ), 8.27 (dd, $\mathrm{J}=$ 1.6 and $9.2 \mathrm{~Hz}, 1 \mathrm{H}, \mathrm{H} 8), 7.87(\mathrm{~d}, \mathrm{~J}=8.5 \mathrm{~Hz}, 2 \mathrm{H}), 7.36$ (dd, $\mathrm{J}=4.4$ and $9.2 \mathrm{~Hz}, 1 \mathrm{H}, \mathrm{H} 7) .{ }^{13} \mathrm{C}$ NMR (100 MHz, $d_{6}$-DMSO) $\delta(\mathrm{ppm}) 144.3,140.5$ $134.1,132.4,127.5$ (q, $J=30.5 \mathrm{~Hz}), 126.3,126.2,125.7,125.5(\mathrm{~m})$, 
124.2 ( $\mathrm{q}, \mathrm{J}=278.5 \mathrm{~Hz}$ ), 117.8. Elemental analysis: calcd (\%) for $\mathrm{C}_{13} \mathrm{H}_{8} \mathrm{~F}_{3} \mathrm{~N}_{3}$ (263.22): C 59.32, H 3.06; found: C 59.27, H 3.18.

Methyl 4-(imidazo[1,2-b]pyridazin-3-yl)benzoate (25): Following the general procedure using methyl 4-chlorobenzoate $(0.170 \mathrm{~g}, 1 \mathrm{mmol})$ and imidazo[1,2-b]pyridazine $(0.131 \mathrm{~g}, 1.1 \mathrm{mmol})$, the residue was purified by flash chromatography on silica gel (pentane- $\mathrm{Et}_{2} \mathrm{O}, 65: 35$ ) to afford the desired compound $25(0.182 \mathrm{~g}, 72 \%)$ as a white solid $\left(\mathrm{mp}=165-167^{\circ} \mathrm{C}\right)$. ${ }^{1} \mathrm{H}$ NMR $\left(400 \mathrm{MHz}, d_{6}\right.$-DMSO) $\delta(\mathrm{ppm}) 8.70(\mathrm{dd}, \mathrm{J}=1.6$ and $4.4 \mathrm{~Hz}, 1 \mathrm{H}$, $\mathrm{H6}$ ), 8.43 (s, 1H, H2), 8.35 (d, $J=8.4 \mathrm{~Hz}, 2 \mathrm{H}$ ), 8.25 (dd, $\mathrm{J}=1.6$ and 9.2 $\mathrm{Hz}, 1 \mathrm{H}, \mathrm{H} 8), 8.07(\mathrm{~d}, \mathrm{~J}=8.4 \mathrm{~Hz}, 2 \mathrm{H}), 7.35(\mathrm{dd}, \mathrm{J}=4.4$ and $9.2 \mathrm{~Hz}, 1 \mathrm{H}$, $\mathrm{H} 7), 3.88(\mathrm{~s}, 3 \mathrm{H}) .{ }^{13} \mathrm{C}$ NMR $\left(100 \mathrm{MHz}, d_{6}\right.$-DMSO) $\delta(\mathrm{ppm}) 165.9,144.1$, 140.6, 134.3, 132.9, 129.5, 128.1, 126.2, 126.1, 125.7, 117.8, 52.1. Elemental analysis: calcd (\%) for $\mathrm{C}_{14} \mathrm{H}_{11} \mathrm{~N}_{3} \mathrm{O}_{2}$ (253.26): C 66.40, H 4.38; found: C $66.49, \mathrm{H} 4.59$.

\begin{abstract}
3-(Imidazo[1,2-b]pyridazin-3-yl)benzonitrile (26): Following the general procedure using 3-chlorobenzonitrile $(0.137 \mathrm{~g}, 1 \mathrm{mmol})$ and imidazo[1,2-b]pyridazine $(0.131 \mathrm{~g}, 1.1 \mathrm{mmol})$, the residue was purified by flash chromatography on silica gel (pentane- $\mathrm{Et}_{2} \mathrm{O}, 65: 35$ ) to afford the desired compound $26(0.159 \mathrm{~g}, 72 \%)$ as a white solid $\left(\mathrm{mp}=194-198{ }^{\circ} \mathrm{C}\right)$. ${ }^{1} \mathrm{H}$ NMR $\left(400 \mathrm{MHz}, d_{6}\right.$-DMSO) $\delta(\mathrm{ppm}) 8.72(\mathrm{dd}, \mathrm{J}=1.7$ and $4.4 \mathrm{~Hz}, 1 \mathrm{H}$, $\mathrm{H6}$ ), 8.65 (s, 1H), 8.52 (ddd, $J=7.7,7.9$ and $8.0 \mathrm{~Hz}, 1 \mathrm{H}$ ), $8.45(\mathrm{~s}, 1 \mathrm{H}$, $\mathrm{H} 2$ ), 8.26 (dd, $J=1.7$ and $9.2 \mathrm{~Hz}, 1 \mathrm{H}, \mathrm{H} 8$ ), 7.84 (ddd, $J=7.7,7.9$ and 8.0 $\mathrm{Hz}, 1 \mathrm{H}), 7.73(\mathrm{~d}, \mathrm{~J}=7.7$ and $7.9 \mathrm{~Hz}, 1 \mathrm{H}), 7.37$ (dd, $\mathrm{J}=4.4$ and $9.2 \mathrm{~Hz}$, $1 \mathrm{H}, \mathrm{H} 7) .{ }^{13} \mathrm{C}$ NMR (100 MHz, $d_{6}$-DMSO) $\delta(\mathrm{ppm}) 144.2,140.4,133.9$, $131.0,130.4,130.0,129.7,129.1,126.2,125.1,118.7,117.9,111.9$ Elemental analysis: calcd (\%) for $\mathrm{C}_{13} \mathrm{H}_{8} \mathrm{~N}_{4}$ (220.24): C 70.90, H 3.66; found: C 71.18, H 3.65 .
\end{abstract}

3-(3-Nitrophenyl)imidazo[1,2-b]pyridazine (27): Following the general procedure using 3-chloronitrobenzene $(0.157 \mathrm{~g}, 1 \mathrm{mmol})$ and imidazo[1,2-b]pyridazine $(0.131 \mathrm{~g}, 1.1 \mathrm{mmol})$, the residue was purified by flash chromatography on silica gel (pentane- $\mathrm{Et}_{2} \mathrm{O}, 55: 45$ ) to afford the desired compound $27(0.173 \mathrm{~g}, 72 \%)$ as a yellow solid $(\mathrm{mp}=170-174$ $\left.{ }^{\circ} \mathrm{C}\right) .{ }^{1} \mathrm{H}$ NMR $\left(400 \mathrm{MHz}, d_{6}-\mathrm{DMSO}\right) \delta(\mathrm{ppm}) 9.13(\mathrm{~s}, 1 \mathrm{H}), 8.75(\mathrm{dd}, \mathrm{J}=$ 1.6 and $4.4 \mathrm{~Hz}, 1 \mathrm{H}, \mathrm{H} 6), 8.57(\mathrm{~d}, \mathrm{~J}=8.0 \mathrm{~Hz}, 1 \mathrm{H}), 8.51(\mathrm{~s}, 1 \mathrm{H}, \mathrm{H} 2), 8.28$ (dd, $J=1.6$ and $9.2 \mathrm{~Hz}, 1 \mathrm{H}, \mathrm{H} 8$ ), $8.21(\mathrm{dd}, J=2.5$ and $8.3 \mathrm{~Hz}, 1 \mathrm{H}$ ), 7.81 (dd, $J=8.0$ and $8.3 \mathrm{~Hz}, 1 \mathrm{H}$ ), $7.38(\mathrm{dd}, \mathrm{J}=4.5$ and $9.2 \mathrm{~Hz}, 1 \mathrm{H}, \mathrm{H} 7) .{ }^{13} \mathrm{C}$ NMR (100 MHz, $d_{6}$-DMSO) $\delta$ (ppm) 148.1, 144.3, 140.5, 134.1, 132.2, $130.2,130.0,126.3,125.0,122.1,119.8,118.0$. Elemental analysis: calcd (\%) for $\mathrm{C}_{12} \mathrm{H}_{8} \mathrm{~N}_{4} \mathrm{O}_{2}$ (240.22): C 60.00, H 3.36; found: $\mathrm{C} 60.23, \mathrm{H}$ 3.57

3-(2-Nitrophenyl)imidazo[1,2-b]pyridazine (28): Following the general procedure using 2-chloronitrobenzene $(0.157 \mathrm{~g}, 1 \mathrm{mmol})$ and imidazo[1,2-b]pyridazine $(0.131 \mathrm{~g}, 1.1 \mathrm{mmol})$, the residue was purified by flash chromatography on silica gel (pentane- $\mathrm{Et}_{2} \mathrm{O}, 55: 45$ ) to afford the desired compound $28(0.156 \mathrm{~g}, 65 \%)$ as a yellow solid $(\mathrm{mp}=180-185$ $\left.{ }^{\circ} \mathrm{C}\right) .{ }^{1} \mathrm{H}$ NMR $\left(400 \mathrm{MHz}, d_{6}\right.$-DMSO) $\delta(\mathrm{ppm}) 8.47(\mathrm{dd}, \mathrm{J}=1.6$ and $4.5 \mathrm{~Hz}$, $1 \mathrm{H}, \mathrm{H} 6), 8.24$ (dd, $\mathrm{J}=1.6$ and $9.3 \mathrm{~Hz}, 1 \mathrm{H}, \mathrm{H} 8), 8.21(\mathrm{~d}, \mathrm{~J}=8.0 \mathrm{~Hz}, 1 \mathrm{H}$ ), 8.12 (s, 1H. H2), 7.91 (ddd, $J=1.5,7.3$ and $8.2 \mathrm{~Hz}, 1 \mathrm{H}$ ), 7.82 (d, $\mathrm{J}=7.3$ $\mathrm{Hz}, 1 \mathrm{H}$ ), 7.76 (ddd, $\mathrm{J}=1.7,7.3$ and $8.2 \mathrm{~Hz}, 1 \mathrm{H}), 7.30(\mathrm{dd}, \mathrm{J}=4.4$ and 9.3 $\mathrm{Hz}, 1 \mathrm{H}, \mathrm{H} 7) .{ }^{13} \mathrm{C}$ NMR (100 MHz, $d_{6}$-DMSO) $\delta(\mathrm{ppm}) 147.6,143.7,139.6$ 134.0, 133.2, 133.1, 130.1, 126.1, 125.0, 122.2, 118.1. Elemental analysis: calcd (\%) for $\mathrm{C}_{12} \mathrm{H}_{8} \mathrm{~N}_{4} \mathrm{O}_{2}$ (240.22): C 60.00, H 3.36; found: C $60.31, \mathrm{H} 3.19$

2-(Imidazo[1,2-b]pyridazin-3-yl)-5-nitrobenzonitrile (29): Following the general procedure using 2-chloro-5-nitrobenzonitrile $(0.183 \mathrm{~g}, 1 \mathrm{mmol})$ and imidazo[1,2-b]pyridazine $(0.131 \mathrm{~g}, 1.1 \mathrm{mmol})$, the residue was purified by flash chromatography on silica gel (pentane- $\mathrm{Et}_{2} \mathrm{O}, 55: 45$ ) to afford the desired compound $29(0.191 \mathrm{~g}, 72 \%)$ as a yellow solid $(\mathrm{mp}=$ 232-234 $\left.{ }^{\circ} \mathrm{C}\right) .{ }^{1} \mathrm{H}$ NMR (400 MHz, $d_{6}$-DMSO) $\delta(\mathrm{ppm}) 8.91(\mathrm{~d}, J=2.6 \mathrm{~Hz}$, $1 \mathrm{H}), 8.71(\mathrm{~d}, \mathrm{~J}=4.5 \mathrm{~Hz}, 1 \mathrm{H}, \mathrm{H} 8), 8.66(\mathrm{dd}, \mathrm{J}=2.5$ and $8.7 \mathrm{~Hz}, 1 \mathrm{H}), 8.42$ (s, 1H, H2), 8.39 (d, J = 9.2 Hz, 1H, H6), 8.35 (dd, J = 1.8 and $9.2 \mathrm{~Hz}, 1 \mathrm{H}$, $\mathrm{H} 6), 7.47$ (dd, $\mathrm{J}=4.5$ and $9.2 \mathrm{~Hz}, 1 \mathrm{H}, \mathrm{H} 7)$. ${ }^{13} \mathrm{C} \mathrm{NMR}\left(100 \mathrm{MHz}, d_{6^{-}}\right.$ DMSO) $\delta$ (ppm) 146.4, 144.5, 140.8, 138.3, 136.6, 136.0, 130.9, 129.2, $127.9,126.5,122.8,119.7,111.4$. Elemental analysis: calcd (\%) for $\mathrm{C}_{13} \mathrm{H}_{7} \mathrm{~N}_{5} \mathrm{O}_{2}$ (265.23): C 58.87, H 2.66; found: C 59.11, H 2.89

6-Chloro-3-(4-nitrophenyl)imidazo[1,2-b]pyridazine (30): Following the general procedure using 4-bromonitrobenzene $(0.200 \mathrm{~g}, 1 \mathrm{mmol})$ and 6-chloroimidazo[1,2-b]pyridazine $(0.169 \mathrm{~g}, 1.1 \mathrm{mmol})$, the residue was purified by flash chromatography on silica gel (pentane- $\mathrm{Et}_{2} \mathrm{O}, 60: 40$ ) to afford the desired compound $\mathbf{3 0}(0.214 \mathrm{~g}, 78 \%)$ as a yellow solid $(\mathrm{mp}=$ 215-218 $\left.{ }^{\circ} \mathrm{C}\right) .{ }^{1} \mathrm{H}$ NMR $\left(400 \mathrm{MHz}, \mathrm{CDCl}_{3}\right) \delta(\mathrm{ppm}) 8.53(\mathrm{~s}, 1 \mathrm{H}), 8.40-8.36$ $(\mathrm{m}, 4 \mathrm{H}), 8.33(\mathrm{~d}, \mathrm{~J}=9.4 \mathrm{~Hz}, 1 \mathrm{H}), 7.52(\mathrm{~d}, \mathrm{~J}=9.4 \mathrm{~Hz}, 1 \mathrm{H})$. This is a known compound and the spectral data are identical to those reported in literature. ${ }^{[11]}$

4-(6-Chloroimidazo[1,2-b]pyridazin-3-yl)benzaldehyde (31): Following the general procedure using 4-bromobenzaldehyde $(0.183 \mathrm{~g}, 1 \mathrm{mmol})$ and 6-chloroimidazo[1,2-b]pyridazine $(0.169 \mathrm{~g}, 1.1 \mathrm{mmol})$, the residue was purified by flash chromatography on silica gel (pentane- $\mathrm{Et}_{2} \mathrm{O}, 60: 40$ ) to afford the desired compound $31(0.211 \mathrm{~g}, 82 \%)$ as a yellow solid $(\mathrm{mp}=$ $\left.145-148{ }^{\circ} \mathrm{C}\right) .{ }^{1} \mathrm{H}$ NMR $\left(400 \mathrm{MHz} \mathrm{CDCl}_{3}\right) \delta(\mathrm{ppm}) 10.05(\mathrm{~s}, 1 \mathrm{H}), 8.28-$ $8.23(\mathrm{~m}, 2 \mathrm{H}), 8.18(\mathrm{~s}, 1 \mathrm{H}), 8.05-7.94(\mathrm{~m}, 3 \mathrm{H}), 7.15(\mathrm{~d}, \mathrm{~J}=9.4 \mathrm{~Hz}, 1 \mathrm{H})$. This is a known compound and the spectral data are identical to those reported in literature. ${ }^{[11]}$

3-(4-Bromophenyl)-6-chloroimidazo[1,2-b]pyridazine (32): Following the general procedure using 1,4-dibromobenzene $(0.236 \mathrm{~g}, 1 \mathrm{mmol})$ and 6-chloroimidazo[1,2-b]pyridazine $(0.169 \mathrm{~g}, 1.1 \mathrm{mmol})$, the residue was purified by flash chromatography on silica gel (pentane- $\mathrm{Et}_{2} \mathrm{O}, 60: 40$ ) to afford the desired compound $32(0.231 \mathrm{~g}, 75 \%)$ as a yellow solid ( $\mathrm{mp}=$ 152-154 $\left.{ }^{\circ} \mathrm{C}\right) .{ }^{1} \mathrm{H}$ NMR $\left(400 \mathrm{MHz}, d_{6}\right.$-DMSO) $\delta(\mathrm{ppm}) 8.37(\mathrm{~s}, 1 \mathrm{H}, \mathrm{H} 2)$ $8.32(\mathrm{~d}, \mathrm{~J}=9.5 \mathrm{~Hz}, 1 \mathrm{H}, \mathrm{H} 8), 8.07$ (d, J = 8.7 Hz, 2H), $7.76(\mathrm{~d}, \mathrm{~J}=8.7 \mathrm{~Hz}$, $2 \mathrm{H}), 7.45$ (d, J = 9.5 Hz, 1H, H7). ${ }^{3} \mathrm{C}$ NMR (100 MHz, $d_{6}$-DMSO) $\delta(p p m)$ 146.5, 138.9, 134.0, 131.8, 128.2, 128.1, 127.0, 126.8, 121.2, 118.9 Elemental analysis: calcd (\%) for $\mathrm{C}_{12} \mathrm{H}_{7} \mathrm{BrClN}_{3}$ (308.56): C 46.71, H 2.29; found: C $46.78, \mathrm{H} 2.35$

Methyl 2-(6-chloroimidazo[1,2-b]pyridazin-3-yl)benzoate (33): Following the general procedure using methyl 2-bromobenzoate $(0.215 \mathrm{~g}$, $1 \mathrm{mmol}$ ) and 6-chloroimidazo[1,2-b]pyridazine $(0.169 \mathrm{~g}, 1.1 \mathrm{mmol})$, the residue was purified by flash chromatography on silica gel (pentane- $\mathrm{Et}_{2} \mathrm{O}$, $55: 45)$ to afford the desired compound $33(0.170 \mathrm{~g}, 59 \%)$ as a yellow solid $\left(\mathrm{mp}=144-147^{\circ} \mathrm{C}\right) .{ }^{1} \mathrm{H}$ NMR $\left(400 \mathrm{MHz}, d_{6}\right.$-DMSO) $\delta(\mathrm{ppm}) 8.29(\mathrm{~d}$, $J=9.5 \mathrm{~Hz}, 1 \mathrm{H}, \mathrm{H} 8), 8.07(\mathrm{~s}, 1 \mathrm{H}, \mathrm{H} 2), 7.96(\mathrm{dd}, \mathrm{J}=1.5$ and $7.7 \mathrm{~Hz}, 1 \mathrm{H}$ ), 7.75 (ddd, $J=1.5,7.4$ and $7.5 \mathrm{~Hz}, 1 \mathrm{H}), 7.68-7.60(\mathrm{~m}, 2 \mathrm{H}), 7.40(\mathrm{~d}, \mathrm{~J}=$ $9.5 \mathrm{~Hz}, 1 \mathrm{H}, \mathrm{H} 7), 3.57$ (s, 3H). ${ }^{13} \mathrm{C}$ NMR (100 MHz, $d_{6}$-DMSO) $\delta(p p m)$ $167.1,145.8,137.9,133.7,132.3,131.8,130.4,129.9,129.2,128.4$ 128.0, 126.8, 118.7, 52.1. Elemental analysis: calcd (\%) for $\mathrm{C}_{14} \mathrm{H}_{10} \mathrm{CIN}_{3} \mathrm{O}_{2}$ (287.70): C 58.45, H 3.50; found: C 58.76, H 3.41.

6-Chloro-3-(naphthalen-1-yl)imidazo[1,2-b]pyridazine (34): Following the general procedure using 1-bromonaphthalene $(0.207 \mathrm{~g}, 1 \mathrm{mmol})$ and 6-chloroimidazo[1,2-b]pyridazine $(0.169 \mathrm{~g}, 1.1 \mathrm{mmol})$, the residue was purified by flash chromatography on silica gel (pentane- $\mathrm{Et}_{2} \mathrm{O}, 45: 55$ ) to afford the desired compound $34(0.213 \mathrm{~g}, 76 \%)$ as a white solid $(\mathrm{mp}=$ $\left.180-183^{\circ} \mathrm{C}\right) .{ }^{1} \mathrm{H}$ NMR $\left(400 \mathrm{MHz}, d_{6}\right.$-DMSO) $\delta(\mathrm{ppm}) 8.36(\mathrm{~d}, J=9.5 \mathrm{~Hz}$, $1 \mathrm{H}, \mathrm{H} 7), 8.12(\mathrm{~d}, \mathrm{~J}=8.0 \mathrm{~Hz}, 1 \mathrm{H}), 8.09(\mathrm{~s}, 1 \mathrm{H}, \mathrm{H} 2), 8.07(\mathrm{~d}, \mathrm{~J}=8.4 \mathrm{~Hz}$, $1 \mathrm{H}), 7.74(\mathrm{dd}, \mathrm{J}=1.5$ and $7.0 \mathrm{~Hz}, 1 \mathrm{H}), 7.68(\mathrm{dd}, J=7.3$ and $8.2 \mathrm{~Hz}, 1 \mathrm{H})$, 7.62-7.55 (m, 2H), 7.5 (ddd, $J=1.6,6.6$ and $8.6 \mathrm{~Hz}, 1 \mathrm{H}), 7.43(\mathrm{~d}, \mathrm{~J}=9.5$ $\mathrm{Hz}, 1 \mathrm{H}, \mathrm{H} 8) .{ }^{13} \mathrm{C}$ NMR (100 MHz, $d_{6}$-DMSO) $\delta(\mathrm{ppm}) 146.4,138.0,135.0$, $133.3,129.6,129.1,128.5,128.1,126.9,126.3,125.5,126.3,125.5$, 125.2, 124.9, 119.0. Elemental analysis: calcd (\%) for $\mathrm{C}_{16} \mathrm{H}_{10} \mathrm{CIN}_{3}$ (279.73): C 68.70, H 3.60; found: C 69.12, H 3.98. 
3-(6-Chloroimidazo[1,2-b]pyridazin-3-yl)quinoline (35): Following the general procedure using 3-bromoquinoline $(0.208 \mathrm{~g}, 1 \mathrm{mmol})$ and 6 chloroimidazo[1,2-b]pyridazine $(0.169 \mathrm{~g}, 1.1 \mathrm{mmol})$, the residue was purified by flash chromatography on silica gel (pentane- $\mathrm{Et}_{2} \mathrm{O}, 20: 80$ ) to afford the desired compound $35(0.174 \mathrm{~g}, 62 \%)$ as a yellow solid $(\mathrm{mp}=$ $\left.218-221^{\circ} \mathrm{C}\right) .{ }^{1} \mathrm{H}$ NMR $\left(400 \mathrm{MHz}, d_{6}\right.$-DMSO) $\delta(\mathrm{ppm}) 9.55(\mathrm{~d}, \mathrm{~J}=2.3 \mathrm{~Hz}$, $1 \mathrm{H}), 9.08(\mathrm{~d}, \mathrm{~J}=2.3 \mathrm{~Hz}, 1 \mathrm{H}), 8.59(\mathrm{~s}, 1 \mathrm{H}), 8.38(\mathrm{~d}, J=9.5 \mathrm{~Hz}, 1 \mathrm{H}, \mathrm{H} 7)$, 8.11-8.06 (m, 2H), 7.82 (ddd, $J=1.5,6.9$ and $8.5 \mathrm{~Hz}, 1 \mathrm{H}), 7.69$ (dd, $J=$ 6.9 and $8.2 \mathrm{~Hz}, 1 \mathrm{H}), 7.52(\mathrm{~d}, \mathrm{~J}=9.5 \mathrm{~Hz}, 1 \mathrm{H}, \mathrm{H} 8) .{ }^{13} \mathrm{C} \mathrm{NMR}(100 \mathrm{MHz}$, $d_{6}$-DMSO) $\delta(\mathrm{ppm}) 148.7,146.7,146.6,129.2,134.5,131.8,130.0$ $128.8,128.5,128.3,127.4,127.2,125.3,121.4,119.4$. Elemental analysis: calcd (\%) for $\mathrm{C}_{15} \mathrm{H}_{9} \mathrm{CIN}_{4}$ (280.72): C 64.18, H 3.23; found: C $64.28, \mathrm{H} 3.45$

6-Chloro-3-(pyridin-3-yl)imidazo[1,2-b]pyridazine (36): Following the general procedure using 3-bromopyridine $(0.157 \mathrm{~g}, 1 \mathrm{mmol})$ and 6 chloroimidazo[1,2-b]pyridazine $(0.169 \mathrm{~g}, 1.1 \mathrm{mmol})$, the residue was purified by flash chromatography on silica gel (pentane- $\mathrm{Et}_{2} \mathrm{O}, 60: 40$ ) to afford the desired compound $36(0.134 \mathrm{~g}, 58 \%)$ as a yellow solid $(\mathrm{mp}=$ 144-147 $\left.{ }^{\circ} \mathrm{C}\right) .{ }^{1} \mathrm{H} \mathrm{NMR}\left(400 \mathrm{MHz}, \mathrm{CDCl}_{3}\right) \delta(\mathrm{ppm}) 9.23(\mathrm{~d}, \mathrm{~J}=2.0 \mathrm{~Hz}, 1 \mathrm{H})$ $8.63(\mathrm{dd}, \mathrm{J}=1.5$ and $4.8 \mathrm{~Hz}, 1 \mathrm{H}), 8.42(\mathrm{td}, \mathrm{J}=1.9$ and $8.0 \mathrm{~Hz}, 1 \mathrm{H}), 8.01$ $(\mathrm{s}, 1 \mathrm{H}), 7.99(\mathrm{~d}, J=9.4 \mathrm{~Hz}, 1 \mathrm{H}), 7.46-7.43(\mathrm{~m}, 1 \mathrm{H}), 7.14(\mathrm{~d}, J=9.4 \mathrm{~Hz}$ $1 \mathrm{H})$. This is a known compound and the spectral data are identical to those reported in literature. ${ }^{[11]}$

2-Bromo-3-(4-fluorophenyl)imidazo[1,2-b]pyridazine (37): 3-(4Fluorophenyl)imidazo[1,2-b]pyridazine (8) $(0.213,1 \mathrm{mmol}), \quad \mathrm{N}$ bromosuccinimide $(0.196 \mathrm{~g}, 1.1 \mathrm{mmol})$ were heated in $5 \mathrm{~mL}$ chloroform at reflux for 12 hour. The solvent was evaporated and the residue was purified by flash chromatography on silica gel (pentane- $\mathrm{Et}_{2} \mathrm{O}, 45: 55$ ) to afford the desired compound $37(0.278 \mathrm{~g}, 95 \%)$ as a white solid $(\mathrm{mp}=$ $172-173{ }^{\circ} \mathrm{C}$ ). ${ }^{1} \mathrm{H}$ NMR $\left(400 \mathrm{MHz}, d_{6}\right.$-DMSO) $\delta(\mathrm{ppm}) 8.57$ (dd, $\mathrm{J}=1.6$ and $4.6 \mathrm{~Hz}, 1 \mathrm{H}, \mathrm{H6}$ ), $8.17(\mathrm{dd}, J=1.6$ and $9.2 \mathrm{~Hz}, 1 \mathrm{H}, \mathrm{H8}), 7.82(\mathrm{dd}, J=$ 5.4 and $8.4 \mathrm{~Hz}, 2 \mathrm{H}), 7.41(\mathrm{t}, J=8.9 \mathrm{~Hz}, 2 \mathrm{H}), 7.35(\mathrm{dd}, J=4.6$ and $9.2 \mathrm{~Hz}$ $1 \mathrm{H}, \mathrm{H} 7) .{ }^{13} \mathrm{C}$ NMR $\left(100 \mathrm{MHz}, d_{6}\right.$-DMSO) $\delta(\mathrm{ppm}) 162.0(\mathrm{~d}, \mathrm{~J}=247.1 \mathrm{~Hz})$ 144.2, 138.2, $131.8(\mathrm{~d}, \mathrm{~J}=8.1 \mathrm{~Hz}), 125.0,124.1,123.0,121.8,118.7$, $115.5(d, J=21.4 \mathrm{~Hz})$. Elemental analysis: calcd (\%) for $\mathrm{C}_{12} \mathrm{H}_{7} \mathrm{BrFN}_{3}$ (292.11): C 49.34, H 2.42; found: C 49.57, H 2.56 .

3-(4-Fluorophenyl)-2-phenylimidazo[1,2-b]pyridazine (38): The reaction of 2-bromo-3-(4-fluorophenyl)imidazo[1,2-b]pyridazine (37) $(0.146 \mathrm{~g}, 0.5 \mathrm{mmol})$, phenylboronic acid $(0.073 \mathrm{~g}, 0.6 \mathrm{mmol})$ and $\mathrm{K}_{3} \mathrm{PO}_{4}$ $(0.212 \mathrm{~g}, 1 \mathrm{mmol})$ at $80{ }^{\circ} \mathrm{C}$ over $15 \mathrm{~h}$ in 1,4-dioxane $(1 \mathrm{~mL})$ in the presence of $\mathrm{PdCl}\left(\mathrm{C}_{3} \mathrm{H}_{5}\right)(\mathrm{dppb})(6 \mathrm{mg}, 0.01 \mathrm{mmol})$ under argon affords, after evaporation of the solvent and purification on silica gel, product 38 $(0.124 \mathrm{~g}, 86 \%)$ as a pale yellow solid $\left(\mathrm{mp}=126-129^{\circ} \mathrm{C}\right) .{ }^{1} \mathrm{H}$ NMR $(400$ $\mathrm{MHz}, d_{6}$-DMSO) $\delta(\mathrm{ppm}) 8.49$ (dd, $J=1.7$ and $\left.4.4 \mathrm{~Hz}, 1 \mathrm{H}, \mathrm{H} 6\right), 8.2$ (dd, $J$ $=1.6$ and $9.2 \mathrm{~Hz}, 1 \mathrm{H}, \mathrm{H} 8), 7.64-7.58(\mathrm{~m}, 4 \mathrm{H}), 7.39-7.26(\mathrm{~m}, 6 \mathrm{H}) .{ }^{13} \mathrm{C}$ NMR (100 MHz, $d_{6}$-DMSO) $\delta(p p m) 162.2(\mathrm{~d}, J=245.3 \mathrm{~Hz}), 143.7,141.8$ $138.3,133.8,132.9(\mathrm{~d}, \mathrm{~J}=8.4 \mathrm{~Hz}), 128.5,127.9,127.7,125.3,125.1(\mathrm{~m})$ $123.4,118.1,115.9(\mathrm{~d}, \mathrm{~J}=22.3 \mathrm{~Hz}$ )

Elemental analysis: calcd (\%) for $\mathrm{C}_{18} \mathrm{H}_{12} \mathrm{FN}_{3}$ (289.31): C 74.73, H 4.18; found: C $74.89, \mathrm{H} 4.07$

3-(4-fluorophenyl)-2-(thiophen-3-yl)imidazo[1,2-b]pyridazine (39): The reaction of 2-bromo-3-(4-fluorophenyl)imidazo[1,2-b]pyridazine (37) $(0.146 \mathrm{~g}, 0.5 \mathrm{mmol})$, thiophen-3-ylboronic acid $(0.077 \mathrm{~g}, 0.6 \mathrm{mmol})$ and $\mathrm{K}_{3} \mathrm{PO}_{4}(0.212 \mathrm{~g}, 1 \mathrm{mmol})$ at $80^{\circ} \mathrm{C}$ over $15 \mathrm{~h}$ in 1,4-dioxane $(1 \mathrm{~mL})$ in the presence of $\mathrm{PdCl}\left(\mathrm{C}_{3} \mathrm{H}_{5}\right)(\mathrm{dppb})(6 \mathrm{mg}, 0.01 \mathrm{mmol})$ under argon affords, after evaporation of the solvent and purification on silica gel, product 39 $(0.131 \mathrm{~g}, 89 \%)$ as a white solid $\left(\mathrm{mp}=186-189^{\circ} \mathrm{C}\right) .{ }^{1} \mathrm{H} \mathrm{NMR}(400 \mathrm{MHz}$, $d_{6}$-DMSO) $\delta(\mathrm{ppm}) 8.46(\mathrm{dd}, J=1.6$ and $4.4 \mathrm{~Hz}, 1 \mathrm{H}, \mathrm{H} 6), 8.17(\mathrm{dd}, J=$ 1.6 and $9.2 \mathrm{~Hz}, 1 \mathrm{H}, \mathrm{H} 8), 7.65-7.60(\mathrm{~m}, 2 \mathrm{H}), 7.55(\mathrm{dd}, J=3.1$ and $5.1 \mathrm{~Hz}$, $1 \mathrm{H}$ ), $7.4(\mathrm{t}, \mathrm{J}=8.8 \mathrm{~Hz}, 2 \mathrm{H}$ ), 7.28 (dd, $J=4.4$ and $9.2 \mathrm{~Hz}, 2 \mathrm{H}$ ), 7.21 (dd, $J$
$=1.0$ and $5.1 \mathrm{~Hz}, 1 \mathrm{H}) \cdot{ }^{13} \mathrm{C}$ NMR $\left(100 \mathrm{MHz}, d_{6}-\mathrm{DMSO}\right) \delta(\mathrm{ppm}) 162.3(\mathrm{~d}$ $J=248.3 \mathrm{~Hz}$ ), 143.6, 138.3, 138.1, 135.1, $133.0(\mathrm{~d}, \mathrm{~J}=9.5 \mathrm{~Hz}), 126.5$ 126.4 , 125.0, $124.9(\mathrm{~m}), 123.2,122.9,118.0,115.9(\mathrm{~d}, J=21.7 \mathrm{~Hz})$. Elemental analysis: calcd (\%) for $\mathrm{C}_{16} \mathrm{H}_{10} \mathrm{FN}_{3} \mathrm{~S}$ (295.34): C 65.07, H 3.41; found: C $65.28, \mathrm{H} 3.56$.

\section{Acknowledgements}

Acknowledgements Text.

Keywords: Palladium • Heterocycles $•$ Aryl Halides $•$ Green Solvents $•$ Phosphine-free

[1] a) F. Kakiuchi, T. Kochi, Synthesis 2008, 3013-3039; b) L. Ackermann R. Vicente, A. R. Kapdi, Angew. Chem. Int. Ed. 2009, 48 9792-9826; c) F. Bellina, R. Rossi, Tetrahedron 2009, 65, 10269-10310; d) X. Chen, K. M. Engle, D.-H. Wang, J.-Q. Yu, Angew. Chem. Int. Ed. 2009, 48, 50945115; e) T. W. Lyons, M. S. Sanford, Chem. Rev. 2010, 110, 11471169; f) E. M. Beck, M. J. Gaunt, Top. Curr. Chem. 2010, 292, 85-121; g) J. Roger, A. L. Gottumukkala, H. Doucet, ChemCatChem 2010, 2, 20-40; h) T. Satoh, M. Miura, Synthesis 2010, 3395-3409; i) C.-L. Sun B.-J. Li, Z.-J. Shi, Chem. Commun. 2010, 46, 677-685; j) S. H. Cho, J. Y. Kim, J. Kwak, S. Chang, Chem. Soc. Rev. 2011, 40, 5068-5083; k) N. Kuhl, M. N. Hopkinson, J. Wencel-Delord, F. Glorius, Angew. Chem. Int. Ed. 2012, 51, 10236-10254; I) B.-J. Li, Z.-J. Shi, Chem. Soc. Rev. 2012, 41, 5588-5598; m) M. C. White, Synlett 2012, 23, 2746-2748; n) J. Yamaguchi, A. D. Yamaguchi, K. Itami, Angew. Chem. Int. Ed. 2012, 51, 8960-9009; o) S. I. Kozhushkov, L. Ackermann, Chem. Sci. 2013, 4 , 886-896; p) M. He, J.-F. Soulé, H. Doucet, ChemCatChem 2014, 6, 1824-1859; q) R. Rossi, F. Bellina, M. Lessi, C. Manzini, Adv. Synth Catal. 2014, 356, 17-117; r) M. Zhang, Y. Zhang, X. Jie, H. Zhao, G. Li, W. Su, Org. Chem. Front. 2014, 1, 843-895; s) M. R. Yadav, R. K. Rit, M. Shankar, A. K. Sahoo, Asian J. Org. Chem. 2015, 4, 846-864; t) K Hirano, M. Miura, Chem. Lett. 2015, 44, 878-873; u) K. Yuan, J.-F. Soulé, H. Doucet, ACS Catal. 2015, 5, 978-991; v) C. B. Bheeter, L. Chen, J.-F. Soulé, H. Doucet, Catal. Sci. Technol. 2016, 6, 2005-2049. a) A. H. M. De Vries, J. M. C. A. Mulders, J. H. M. Mommers, H. J. W. Henderickx, J. G. De Vries, Org. Lett. 2003, 5, 3285-3288; b) M. T. Reetz, J. G. De Vries, Chem. Commun. 2004, 1559-1563; c) J. G. de Vries, Dalton Trans. 2006, 421-429.

[3] a) X. Wang, D. V. Gribkov, D. Sames, J. Org. Chem. 2007, 72, 1476 1479; b) J. J. Dong, J. Roger, F. Pozgan, H. Doucet, Green Chem. 2009, 11, 1832-1846; c) D. Zhao, W. Wang, S. Lian, F. Yang, J. Lan, J. You, Chem. Eur. J. 2009, 15, 1337-1340; d) H. Y. Fu, L. Chen, H. Doucet, J. Org. Chem. 2012, 77, 4473-4478; e) P. Ehlers, A. Petrosyan, J. Baumgard, S. Jopp, N. Steinfeld, T. V. Ghochikyan, A. S. Saghyan, C. Fischer, P. Langer, ChemCatChem 2013, 5, 2504-2511; f) S. Islam, I. Larrosa, Chem. Eur. J. 2013, 19, 15093-15096; g) S. Hayashi, T. Koizumi, Polym. Chem. 2015, 6, 5036-5039; h) W. Hagui, N. Besbes, E. Srasra, J.-F. Soulé, H. Doucet, RSC Adv. 2016, 6, 17110-17117.

[4] a) K. Alfonsi, J. Colberg, P. J. Dunn, T. Fevig, S. Jennings, T. A Johnson, H. P. Kleine, C. Knight, M. A. Nagy, D. A. Perry, M. Stefaniak, Green Chem. 2008, 10, 31-36; b) C. Capello, U. Fischer, K Hungerbuhler, Green Chem. 2007, 9, 927-934; c) R. K. Henderson, C. Jimenez-Gonzalez, D. J. C. Constable, S. R. Alston, G. G. A Inglis, G. Fisher, J. Sherwood, S. P. Binks, A. D. Curzons, Green Chem. 2011, 13, 854-862.

[5] a) P. Arockiam, V. Poirier, C. Fischmeister, C. Bruneau, P. H. Dixneuf, Green Chem. 2009, 11, 1871-1875; b) J. J. Dong, J. Roger, C. Verrier, T. Martin, R. Le Goff, C. Hoarau, H. Doucet, Green Chem. 2010, 12, 2053-2063; c) C. Fischmeister, H. Doucet, Green Chem. 2011, 13, 741753; d) A. Hfaiedh, K. Yuan, H. Ben Ammar, B. Ben Hassine, J.-F. Soulé, H. Doucet, ChemSusChem 2015, 8, 1794-1804.

[6] a) P. Tundo, M. Selva, Acc. Chem. Res. 2002, 35, 706-716; b) M. Selva Pure Appl. Chem. 2007, 79, 1855-1867; c) T. Sakakura, K. Kohno, Chem. Commun. 2009, 1312-1330; d) B. Schäffner, F. Schäffner, S. P. Verevkin, A. Börner, Chem. Rev. 2010, 110, 4554-4581; e) A. E. DiazAlvarez, J. Francos, B. Lastra-Barreira, P. Crochet, V. Cadierno, Chem. Commun. 2011, 47, 6208-6227; f) C. Samori, M. Basaglia, S. Casella, L. Favaro, P. Galletti, L. Giorgini, D. Marchi, L. Mazzocchetti, C. Torri, E. Tagliavini, Green Chem. 2015, 17, 1047-1056.

[7] a) K. Beydoun, H. Doucet, ChemSusChem 2011, 4, 526-534; b) S. Bensaid, H. Doucet, C. R. Chim. 2014, 17, 1184-1189.

[8] a) L. Ackermann, R. Vicente, Org. Lett. 2009, 11, 4922-4925; b) S Bensaid, N. Laidaoui, D. El Abed, S. Kacimi, H. Doucet, Tetrahedron Lett. 2011, 52, 1383-1387. 
[9] a) G. L. Turner, J. A. Morris, M. F. Greaney, Angew. Chem., Int. Ed. Engl. 2007, 46, 7996-8000; b) S. A. Ohnmacht, P. Mamone, A. J. Culshaw, M. F. Greaney, Chem. Commun. 2008, 1241-1243; c) P. B Arockiam, C. Fischmeister, C. Bruneau, P. H. Dixneuf, Angew. Chem. Int. Ed. Engl. 2010, 49, 6629-6632; d) Y.-X. Su, Y.-H. Deng, T.-T. Ma, Y.-Y. Li, L.-P. Sun, Green Chem. 2012, 14, 1979-1981.

[10] K.-i. Kusakabe, N. Ide, Y. Daigo, T. Itoh, T. Yamamoto, H. Hashizume, K. Nozu, H. Yoshida, G. Tadano, S. Tagashira, K. Higashino, Y. Okano, Y. Sato, M. Inoue, M. Iguchi, T. Kanazawa, Y. Ishioka, K. Dohi, Y. Kido, S. Sakamoto, S. Ando, M. Maeda, M. Higaki, Y. Baba, Y. Nakamura, J. Med. Chem. 2015, 58, 1760-1775.

[11] A. El Akkaoui, S. Berteina-Raboin, A. Mouaddib, G. Guillaumet, Eur. J. Org. Chem. 2010, 2010, 862-871.

[12] a) F. Shibahara, E. Yamaguchi, T. Murai, J. Org. Chem. 2011, 76, 2680-2693; b) S. Suzuki, Y. Segawa, K. Itami, J. Yamaguchi, Nat. Chem. 2015, 7, 227-233.

[13] A. Takfaoui, R. Touzani, J.-F. Soulé, P. H. Dixneuf, H. Doucet, Asian J. Org. Chem. 2015, 4, 1085-1095.

[14] a) A. L. Gottumukkala, H. Doucet, Eur. J. Inorg. Chem. 2007, 3629 3632; b) L. Ackermann, R. Vicente, R. Born, Adv. Synth. Catal. 2008 350, 741-748; c) D. Roy, S. Mom, M. Beaupérin, H. Doucet, J. C Hierso, Angew. Chem., Int. Ed. Engl. 2010, 49, 6650-6654; d) P. V.
Kumar, W.-S. Lin, J.-S. Shen, D. Nandi, H. M. Lee, Organometallics 2011, 30, 5160-5169; e) S. Mom, M. Beauperin, D. Roy, S. Royer, R. Amardeil, H. Cattey, H. Doucet, J. C. Hierso, Inorg. Chem. 2011, 50 , 11592-11603; f) L. Theveau, C. Verrier, P. Lassalas, T. Martin, G. Dupas, O. Querolle, L. Van Hijfte, F. Marsais, C. Hoarau, Chem. Eur. J. 2011, 17, 14450-14463; g) H. Cao, Y. Lin, H. Zhan, Z. Du, X. Lin, Q.-M. Liang, H. Zhang, RSC Adv. 2012, 2, 5972-5975; h) D. Ghosh, H. M. Lee, Org. Lett. 2012, 14, 5534-5537; i) B. Liu, Z. Wang, N. Wu, M. Li, J. You, J. Lan, Chem. Eur. J. 2012, 18, 1599-1603; j) D. Roy, S. Mom, S Royer, D. Lucas, J.-C. Hierso, H. Doucet, ACS Catal. 2012, 2, 10331041; k) X.-B. Shen, Y. Zhang, W.-X. Chen, Z.-K. Xiao, T.-T. Hu, L.-X. Shao, Org. Lett. 2014, 16, 1984-1987.

[15] S. Sahnoun, S. Messaoudi, J.-D. Brion, M. Alami, Org. Biomol. Chem. 2009, 7, 4271-4278.

[16] A. L. Gottumukkala, H. Doucet, Eur. J. Inorg. Chem. 2007, 2007, 36293632 .

[17] T. Cantat, E. Génin, C. Giroud, G. Meyer, A. Jutand, J. Organomet. Chem. 2003, 687, 365-376. 


\section{Supporting Information}

Such materials are peer reviewed and may be re-organized for online delivery, but are not copy-edited or typeset. Technical support issues arising from supporting information (other than missing files) should be addressed to the authors.

\section{Filename}

asia201600827-sup-0001-misc_information.pdf
Format Size Description

8914K Supplementary 